\title{
Towards ultimate bandwidth photon sources based on Compton backscattering: Design constraints due to nonlinear effects
}

\author{
Aurélien Martens®, Fabian Zomer, Manar Amer@, Loïc Amoudry, Kevin Cassou®, \\ Kevin Dupraz $\odot$, and Daniele Nutarelli॰ \\ Université Paris-Saclay, CNRS/IN2P3, IJCLab, 91405 Orsay, France
}

(Received 16 February 2021; accepted 23 August 2021; published 15 September 2021)

\begin{abstract}
Nuclear resonance fluorescence experiments typically require high rates of monochromatic photons due to the narrow linewidth of these resonances. Inverse Compton scattering sources are used to perform these experiments. Their intrinsic excellent monochromaticity is however spoiled by a variety of unavoidable imperfections related to the electron and laser beams. Some projects aim at reaching one per-mille of energy bandwidth, which requires attaining excellent brilliance of the electron beam but also a careful optimization of the laser-beam parameters. In particular, in such a situation, a careful accounting for the nonlinearities induced by a relatively large laser energy has to be considered. In this article, we revisit these nonlinearities with a quantum viewpoint with the goal to provide analytical expressions that can be employed for a very fast optimization of the performance of the source. These expressions were benchmarked against the CAIN event generator with an excellent accuracy in the parameters hypervolume that is of interest in this context. We also show that previously published expression often used to include laser nonlinearities in analytical bandwidth expressions significantly depart from the detailed CAIN simulations. The obtained expression are further used to optimize designs similar to those considered in on-going projects.
\end{abstract}

DOI: 10.1103/PhysRevAccelBeams.24.091601

\section{INTRODUCTION}

Several facilities are currently providing $\gamma$-rays with high-photon rates, low bandwidth, energy tunable, and high polarization mostly for nuclear physics applications and nondestructive analysis [1,2]. Projects are also being implemented all around the world to provide similar sources with improved performances [3-6]. These sources are exploiting the energy up-shifting of photons scattered off relativistic electrons by the Compton process. A typical application of such inverse Compton scattering sources (ICS) is the nuclear resonance fluorescence (NRF) which exhibits very narrow linewidths that are experiencing thermal broadening at room temperature, such that the effective width of these resonances is about $1 \mathrm{eV}$ [7]. To this respect, it is an essential asset for ICS to reduce as much as possible the bandwidth of the emitted radiation while maximizing the photon rate. It justifies that in some projects, the advertised target is to reach the unprecedented $0.1 \%$ bandwidth [8], that we dub ultimate in this article.

\footnotetext{
*aurelien.martens@ijclab.in2p3.fr
}

Published by the American Physical Society under the terms of the Creative Commons Attribution 4.0 International license. Further distribution of this work must maintain attribution to the author(s) and the published article's title, journal citation, and DOI.
The unequivocal relation between the energy of the scattered photons and their emission angle with respect to the electron line of flight may allow to obtain extremely narrow photon beam bandwidths by selecting a small fraction of the photon yield. However, this relation is smeared by various imperfections related to the electron and laser beam phase spaces [9-13]. In particular, electron beam transverse emittance induces a spread in the reference axis of the emitted photons and thus at a given measurement point a spread in energy toward smaller energy values. The energy spread of the electron beam implies an unavoidable spread in the maximum energy of the emitted photons. In particular, a $0.1 \%$ energy spread of the electron beam approximately translates into a $0.2 \%$ energy spread of the photon beam since the scattered photon energy approximately scales as the square of the electron beam energy. The laser beam spectrum width also affects the spectral broadening of the scattered photon energy. More detailed and accurate expressions of scattered photon energy also show that it depends on the crossing angle $\theta_{i}$ between the laser and electron beams. The divergence of the laser beam, i.e. its transverse momentum spread, thus contributes to the overall bandwidth of the scattered photon beam. This dependence is however smooth for small crossing angles.

Various schemes for producing photon beams by inverse Compton scattering are often considered. In most cases, the emittance and the energy spread of the electron beam are 
limiting factors. When the goal is to provide high rates of photons, it is natural to consider building an electron ring coupled to a high-repetition rate, high-power laser system, as for instance an optical cavity $[14,15]$. However, the impact of the Compton scattering on the electron beam dynamics in the ring must be accounted for $[4,16]$ and may contribute to particularly worsen the energy spread. For instance, in configurations studied in Ref. [4], the energy spread was found to increase by an order of magnitude, reaching several per-mille. This may prevent ultimate photon beam bandwidths to be generated by this mean. An other approach consist in using an energy recovery linac which may allow to combine the excellent electron beam properties of a linac along with high-repetition rates related to the continuous operation of the source [17]. However, the demonstration of small emittances, as those expected with pulsed rf photoguns $[8,18,19]$ still needs to be done, to the best of our knowledge. Given the higher rates of scattered photons that can be achieved with this technology, it is nevertheless considered in several projects [20,21].

Linacs operated in pulsed mode represent an interesting alternative. Recent advances in the operation [22] and design [23] of burst mode optical cavities to significantly enhance the laser power available for collisions with several hundreds of electron bunches seems an interesting avenue to consider. It must be pointed out that a similar accelerator technology was at the basis of several modern ICS being considered $[3,8]$ but with a high-energy optical circulator instead of a resonant optical cavity [24]. The suggestion that extremely small emittance electron beams with typically $100 \mathrm{pC}$ bunch charge and $55 \mathrm{nmrad}$ may be produced with pulsed rf, cryogenically cooled photoguns [19] opens the path toward ultimate bandwidth ICS. For an ICS based on this technology combined with optimized burst mode optical cavity, the so-called nonlinear contribution due to the relatively large laser pulse energy must then be precisely accounted for. Such an additional contribution to the ICS bandwidth is usually classically computed and estimated with simulations of the classical electron trajectory in the laser field, see for instance Refs. [9,25-28] or requires expensive calculations [29,30].

In order to quickly estimate the performance of an ICS, it is often convenient at a design stage to employ approximate analytical or semianalytical formula to quantify the effect of the various contributions that deteriorate the photon beam quality. To this extent, the traditional approach was to consider a classical viewpoint [27], validated by means of classical trajectories simulations. More recently the KleinNishina cross section was used to derive an approximate expression of both the ICS bandwidth and spectral density, see Refs. [11,31] and reference therein. It must be noted that the so-called nonlinear term is however obtained through a classical treatment [26]. It is also noteworthy that both classical and quantum simulation codes have been compared with high-intensity laser ICS data taken at Helmholtz Zentrum Dresden Rossendorf [32,33].

Despite much work already exists in the literature with a classical viewpoint, it is enlightening to adopt a quantum electrodynamics (QED) viewpoint to address the question of the impact of nonlinear terms. Cross sections are introduced in Sec. II. Then currently used approximate analytical formula to optimize ICS are introduced in Sec. III. We then show in this paper that the QED viewpoint allows to disentangle several types of contributions unambiguously and that relatively simple approximate formula are obtained to asses the ICS bandwidth and spectral density in Sec. IV. These are benchmarked against an event generator dedicated to electron-photon interaction and that embeds all the effects that are discussed here. This benchmarking is made at high-laser intensity to ease the comparisons. We finally apply in Sec. V the derived expressions to the optimization of an ICS that could be based on high-brightness electron beams as proposed in Ref. [19] for instance by employing an optimized burst mode optical cavity [23] or beams that are expected to be delivered for the PERLE project [21]. This is where the design of a per-mille bandwidth photon source is targeted.

\section{NONLINEAR SCATTERING BETWEEN PHOTONS AND ELECTRONS}

In this section, we first review several effects related to the intensity of the laser beam that are affecting performances of ICS. We describe three effects that need to be accounted for in the design of ultimate ICS. First, we start by some statement about higher QED corrections that turn out to be negligible. We then describe and roughly estimate the effect related to the fact that when the single electron scattering probability approaches unity, it is likely that a single electron scatters several times off laser photons. Second, we include a description and rough estimation of the influence of the presence of a strong-field due to the laser itself at the interaction point. Finally, we provide cross sections for the process where multiple photons are absorbed while a single one is emitted.

We start by emphasizing that, in this paper, we neglect any higher order QED corrections to the linear single Compton scattering [34-39]. In particular radiative corrections [36,37] and double Compton scattering [38,39], the emission of two photons when a single one is absorbed are neglected. The relative magnitude of the latter with respect to single Compton scattering scales as $\mathcal{R}=\alpha X_{0}^{2} / 4$, where $\alpha \approx 1 / 137$ is the fine-structure constant. With typical parameters of an ICS for production of $\gamma$-rays in the few $\mathrm{MeV}$ range, $X_{0} \approx 5 \times 10^{-3}$ thus $\mathcal{R} \approx 2 \times 10^{-7} \quad[38,39]$. This process was also studied in the presence of a very strong laser field [30], with much larger intensity than that considered here. Despite the lack of precise numerical estimation of the magnitude of this effect in the typical situation where we stand, these previous studies suggest 
that this contribution can be fairly neglected, also when dealing with ultimate ICS. They may however need to be accounted for when considering the interaction of very intense lasers [30], where coherent emission of photons may not be negligible. It must be emphasized that the distinction between radiative corrections and multiple Compton scattering is somewhat arbitrary in the sense that, for instance, infrared divergences of the radiative corrections to the single Compton scattering are exactly canceled by the destructive interference of double Compton scattering diagrams when the energy of the second photon vanishes [40]. These magnitude of these contributions is considerably increasing in the scattering of much higher energy beams [34-37], where they can reach a few permille for several tens of $\mathrm{GeV}$ electrons.

The single process where $n$-photon absorption and $n$ photon emission occur would contribute to $\alpha^{2(n-1)}$ higher order corrections to the single Compton process, and is also neglected for a similar reason. However, it is possible that two (or more) totally independent scatterings of the electron during its long travel through the laser pulse contribute significantly. This plural-scattering effect [41] is estimated in Sec. II. Eventually one high-order process that we will still include in the following treatment is the absorption of few photons and emission of a single one. This multiple photon absorption process induce generation of harmonics that must be considered further and precisely estimated. It is often simply dubbed nonlinear Compton scattering, despite several nonlinearities can be identified. We aim at clarifying their respective contributions to an ICS performance in this paper.

\section{A. Cross sections in the single or multiple photon absorption regime}

In an intense laser field, it is likely that an incident electron absorbs several $n \geq 1$ photons and emits a single one, $e^{-}+$ $n \gamma \rightarrow e^{-}+\gamma$. This multiphoton absorption process induces the generation of harmonics of the scattered photon energy. We distinguish the asymptotic situation where the laser is either purely circularly or purely linearly polarized. The cross sections for multiple photon absorption were derived in Refs. [42-44] and can also be found in Refs. [29,41].

\section{Electron mass in the strong field}

The propagation of an electron in the strong field of a nearly counter-propagating laser pulse may absorb and emit an arbitrary number of photons without suffering net scattering. This contribution intervenes in an equivalent manner as virtual diagrams in QED, which means that it contributes to the electron self-energy. Thus, the electron mass is renormalized to a different numerical value depending on the laser intensity $I$ [45]. A comprehensive historical review may be found in Ref. [46]. The effective electron mass in the intense laser field reads

$$
m=m_{e} \sqrt{1+\eta^{2}},
$$

where

$$
\eta^{2}=\frac{e^{2} \lambda^{2} \mu_{0} I}{4 \pi^{2} m_{e}^{2} c^{3}} \approx 3.65 \times 10^{-19} I\left[\mathrm{~W} / \mathrm{cm}^{2}\right] \lambda[\mu \mathrm{m}]^{2},
$$

and where $e$ is the elementary charge, $\lambda$ is the laser wavelength, $m_{e}$ is the electron rest mass, $c$ is the speed of light in vacuum, and $\mu_{0}$ is the permeability of free space. This parameter $\eta^{2}$ is also often denoted $a_{0}^{2}$ or $\xi^{2}$ in the literature.

\section{Purely circular laser polarization}

The differential cross section with a purely circularly polarized laser and an unpolarized electron beam reads $[29,41]$

$$
\begin{aligned}
\frac{d \sigma_{n}^{\text {circ }}}{d E_{\gamma}}= & \frac{2 \pi r_{e}^{2}}{X_{0} \eta^{2} E_{e}}\left(-4 J_{n}^{2}\left(z_{n}\right)+\eta^{2}\left(\frac{X}{Y}+\frac{Y}{X}\right)\right. \\
& \left.\times\left(J_{n-1}^{2}\left(z_{n}\right)+J_{n+1}^{2}\left(z_{n}\right)-2 J_{n}^{2}\left(z_{n}\right)\right)\right),
\end{aligned}
$$

where $n$ denotes the number of photons absorbed in the $e^{-}+n \gamma \rightarrow e^{-}+\gamma$ process. One defines here

$$
X=\frac{2 p \cdot k}{m^{2} c^{4}}=\frac{X_{0}}{1+\eta^{2}} \quad \text { and } \quad Y=\frac{2 p \cdot k^{\prime}}{m^{2} c^{4}}
$$

where the dot designates the four-momentum scalar product, $p(k)$ is the incoming electron (photon) four-momentum, $k^{\prime}$ is the four-momentum of the emitted photon, $E_{e}$ denotes the electron energy, and $r_{e}$ the so-called classical electron radius. It can be shown that the quantity $z_{n}$ is such that

$$
z_{n}^{2}=\frac{4 \eta^{2}}{1+\eta^{2}} \frac{X-Y}{X Y}\left(n-\frac{X-Y}{X Y}\right) .
$$

We define $r$ such that $r X=\frac{\tilde{Y}}{1-\tilde{Y}}$ and $\tilde{Y}=E_{\gamma} / E_{e}$. It can be shown that $Y \approx X(1-\tilde{Y})[41,47]$ when $\gamma \gg 1$. Employing this approximation,

$$
z_{n}^{2} \approx \frac{4 \eta^{2}}{1+\eta^{2}} r(n-r) .
$$

In the weakly nonlinear regime, $\eta^{2} \ll 1$, these differential cross sections are expanded in series against the parameter $\eta^{2}$ and the Bessel functions are approximated as $J_{k}\left(z_{n}\right) \approx \frac{1}{k !} \frac{z_{n}^{k}}{2^{k}}$, such that

$$
\begin{aligned}
\frac{d \sigma_{n}^{\mathrm{circ}}}{d E_{\gamma}} \approx & \frac{2 \pi r_{e}^{2}}{X_{0} \eta^{2} E_{e}}\left(-4 \frac{z_{n}^{2 n}}{2^{2 n}(n !)^{2}}+\eta^{2}(1-\tilde{Y}\right. \\
& \left.\left.+\frac{1}{1-\tilde{Y}}\right) \frac{z_{n}^{2(n-1)}}{2^{2(n-1)}((n-1) !)^{2}}\right) .
\end{aligned}
$$


For $n=1$, it reduces to

$$
\frac{d \sigma_{1}^{\mathrm{circ}}}{d \tilde{Y}} \approx \frac{2 \pi r_{e}^{2}}{X_{0}}\left(-\frac{4}{1+\eta^{2}} r(1-r)+1-\tilde{Y}+\frac{1}{1-\tilde{Y}}\right),
$$

which is the linear Compton cross section $\sigma_{C}$ of Eq. (23) after integration of $\tilde{Y}$ in its valid kinematic range $\left[0 ; \tilde{Y}_{\max }\right]$, with $\tilde{Y}_{\max }=\frac{X}{1+X}$, and doing the replacement $\eta^{2} \rightarrow 0$. It must be however noted that this equation provides a modification of the Klein-Nishina cross section with the first-order contribution due to the relatively intense laser field $\eta^{2}$. This modification will be used in the next part of this paper. We also emphasizes here that instead of providing the truncated expansion of the expression to a given order in $\eta^{2}$ we provide an expression that is equivalent at that order but is more compact, retaining $\left(1+\eta^{2}\right)$ terms at the denominator, for instance. This remark is valid throughout the paper and does not affect significantly the numerical results. For $n=2$, it reduces to

$\frac{d \sigma_{2}^{\mathrm{circ}}}{d \tilde{Y}} \approx \frac{2 \pi r_{e}^{2}}{X_{0}} \frac{\eta^{2}}{1+\eta^{2}} r(2-r)\left(-\frac{r(2-r)}{1+\eta^{2}}+1-\tilde{Y}+\frac{1}{1-\tilde{Y}}\right)$,

where one clearly sees that this contribution comes as a firstorder correction in $\eta^{2}$ to the total scattering probability.

\section{Purely linear laser polarization}

The Compton cross section for a purely linearly polarized laser beam shows some dependence on the azimuthal angle $\theta$ of the scattered photon. However, in practice apertures that are usually implemented in the scattered photon beam to select a narrow bandwidth photon beam are following, or at least approximately [3], cylindrical symmetry. This prescription allows to further simplify the cross section formula for multiphoton absorption $[29,41,48]$

$$
\begin{aligned}
\frac{d \sigma_{n}^{\operatorname{lin}}}{d E}= & \frac{16 r_{e}^{2}}{X_{0} \eta^{2} E_{e}} \int_{0}^{\frac{\pi}{2}} d \phi\left(-A_{0}^{2}\left(n, a_{n}, b\right)\right. \\
& +\eta^{2}\left(\frac{X}{Y}+\frac{Y}{X}\right)\left(A_{1}^{2}\left(n, a_{n}, b\right)\right. \\
& \left.\left.-A_{0}\left(n, a_{n}, b\right) A_{2}\left(n, a_{n}, b\right)\right)\right)
\end{aligned}
$$

where

$$
\begin{aligned}
& A_{k}\left(n, a_{n}, b\right) \\
& =\frac{1}{\pi} \int_{0}^{\pi} d \nu \cos ^{k} \nu \cos \left[\left(a_{n}+2 b \cos \nu\right) \sin \nu-n \nu\right],
\end{aligned}
$$

$a_{n}=\sqrt{2} \cos \phi z_{n}$ and $b=-\eta^{2} r / 2$. Expanding these integrals in powers of $\eta$ provides

$$
\frac{d \sigma_{1}^{\operatorname{lin}}}{d \tilde{Y}} \approx \frac{2 \pi r_{e}^{2}}{X_{0}}\left(1-\tilde{Y}+\frac{1}{1-\tilde{Y}}-\frac{4}{1+\eta^{2}} r(1-r)\right),
$$

which, as expected, after integration over the azimuthal angle $\phi$, equates that for a circularly polarized laser beam, see Eq. (7). For $n=2$, the differential cross section reads

$$
\begin{aligned}
\frac{d \sigma_{2}^{\operatorname{lin}}}{d \tilde{Y}} \approx & \frac{\pi r_{e}^{2} \eta^{2} r}{2 X_{0}}\left[\left(1-\tilde{Y}+\frac{1}{1-\tilde{Y}}\right)\left(1+\frac{2(2-r)}{1+\eta^{2}}\right)\right. \\
& \left.-2 r\left(1-4 \frac{2-r}{1+\eta^{2}}+\frac{6(2-r)^{2}}{\left(1+\eta^{2}\right)^{2}}\right)\right] .
\end{aligned}
$$

For $n=3$, the differential cross section reads

$$
\begin{aligned}
\frac{d \sigma_{3}^{\operatorname{lin}}}{d \tilde{Y}} \approx & \frac{\pi r_{e}^{2} \eta^{4} r^{2}}{8 X_{0}\left(1+\eta^{2}\right)^{2}}\left[\left(1-\tilde{Y}+\frac{1}{1-\tilde{Y}}\right)\right. \\
& \times\left(19-2 r(6-r)+2 \eta^{2}+\eta^{4}\right) \\
& -\frac{4 r(3-r)}{9\left(1+\eta^{2}\right)}(45-2 r(21-5 r) \\
& \left.\left.-18 \eta^{2}(2-r)+9 \eta^{4}\right)\right] .
\end{aligned}
$$

Higher order multiphoton absorption cross section can be computed in a similar manner, but we will see that numerically they do not significantly contribute for the use cases that are investigated in this article.

\section{ANALYTICAL MODEL}

We follow the strategy that has been developed in Refs. [11,49] and references therein. An analytical model for inverse Compton scattering in a narrow emission cone around the electron beam axis is given. It provides both the number of photons emitted in average in a given circular aperture and their energy spread assuming that the collision between the electron and laser beam is head-on and that the electron beam is cylindrical. No effect of electron beam divergence, or hourglass, is included. For simplicity we will not consider an elliptic beam, though this effect can be accounted for [50]. It however considers momentum spreads (transverse and longitudinal) of the electron beam. The laser-beam finite divergence is included in the model as well as its finite spectrum assuming that the laser beam is at the Fourier limit i.e. free of any kind of chirp. A nonlinear term in laser intensity is included as an effective term in the formula. We propose to review this latter term in this article.

The total rate of backscattered photons $\mathcal{N}_{\text {tot }} \approx \mathcal{L} \sigma_{C}$ is approximately the product of the Compton cross section $\sigma_{C}$ and the space-time overlap of the electron and laser beams embedded in a luminosity term $\mathcal{L}$ that reads [51]

$$
\mathcal{L}=f_{\text {rep }} \frac{\mathcal{Q}}{e} \frac{U \lambda}{h c} \mathcal{L}_{0}\left(\sigma_{x ; l}, \sigma_{y ; l}, \sigma_{z ; l}\right)
$$

with 


$$
\begin{aligned}
\mathcal{L}_{0}\left(\sigma_{x ; l}, \sigma_{y ; l}, \sigma_{z ; l}\right)= & \frac{1}{2 \pi \sqrt{\sigma_{y ; e}^{2}+\sigma_{y ; l}^{2}}} \\
& \times \frac{1}{\sqrt{\sigma_{x ; e}^{2}+\sigma_{x ; l}^{2}+\left(\sigma_{z ; e}^{2}+\sigma_{z ; l}^{2}\right) \tan ^{2}\left(\frac{\theta_{i}}{2}\right)}}
\end{aligned}
$$

under the assumptions of collimated Gaussian beams at the interaction point, with vanishing six dimensional-phase space correlations; and where, $f_{\text {rep }}$ is the effective [52] repetition rate of the interaction, $\mathcal{Q}$ denotes the charge of a single electron bunch, $e$ is the elementary charge, $U$ is the energy stored in a laser pulse, $\lambda$ is the laser wavelength, $\theta_{i}$ is the crossing angle assumed to be purely in the horizontal plane, and $\sigma_{a ; b}$ is the rms width of the $(b=e)$ electron and ( $b=l$ ) laser beams in the $a=x, y, z$ horizontal, vertical, and longitudinal directions, respectively. These approximations are often meaningful. It must be emphasized that in case hourglass effect may be of concern, it can be numerically computed or analytically approximated [53]. Also assuming cylindrical beams, $\mathcal{L}_{0}$ reduces to

$$
\mathcal{L}_{0}\left(\sigma_{x ; l}, \sigma_{x ; l}, \sigma_{z ; l}\right)=\frac{1}{2 \pi\left(\sigma_{x ; e}^{2}+\sigma_{x ; l}^{2}\right)} \frac{1}{\sqrt{1+\frac{\sigma_{z e}^{2}+\sigma_{z l}^{2}}{\sigma_{x ; e}^{2}+\sigma_{x ; l}^{2}} \tan ^{2}\left(\frac{\theta_{i}}{2}\right)}} .
$$

The rate of photons $\mathcal{N}_{\theta_{\max }}=R\left(\theta_{\max }\right) \mathcal{N}_{\text {tot }}$ actually produced within a cone defined by a small opening angle $\theta_{\max } \ll 1 / \gamma$ is further reduced compared to the total rate of backscattered photons $\mathcal{N}_{\text {tot }}$ by a multiplicative factor

$$
R\left(\theta_{\max }\right) \simeq \frac{3}{2} \frac{\gamma^{2} \theta_{\max }^{2}}{1+2 \gamma^{2} \theta_{\max }^{2}}
$$

under the additional approximation that the recoil of the electron is negligible [11,13].

Denoting by $E_{e}$ the electron energy and $E_{\lambda}=h \nu$ the energy of a laser photon, the scattered photon energy reads approximately $E_{\gamma} \approx 4 \frac{E_{e}^{2} E_{\lambda}}{m_{e}^{2} c^{4}+E_{e}^{2} \theta^{2}}$, where $\theta$ is the scattering angle of the photon with respect to the line of flight of the electron and $m_{e}$ its mass. The relative spectral width of the produced photon beam is shown [10-12] to follow

$$
\begin{aligned}
\frac{\sigma_{E_{\gamma}}^{2}}{\left\langle E_{\gamma}\right\rangle^{2}}= & \frac{\left\langle E_{\gamma}^{2}\right\rangle-\left\langle E_{\gamma}\right\rangle^{2}}{\left\langle E_{\gamma}\right\rangle^{2}} \\
\simeq & \left(\frac{\Psi_{0}^{2}}{\sqrt{12}\left(1+X_{0}+\frac{\Psi_{0}^{2}}{2}\right)}+\frac{\sqrt{2}}{1+X_{0}} \sqrt{\frac{\epsilon_{n, x}^{2}}{\sigma_{x e}^{2}}+\frac{\epsilon_{n, y}^{2}}{\sigma_{y e}^{2}}}\right)^{2} \\
& +\left(\frac{2+X_{0}}{1+X_{0}+\Psi_{0}^{2}} \frac{\sigma_{E_{e}}}{E_{e}}\right)^{2}+\left(\frac{1+\Psi_{0}^{2}}{1+X_{0}+\Psi_{0}^{2}} \frac{\sigma_{E_{\lambda}}}{E_{\lambda}}\right)^{2} \\
& +\left(\frac{M^{2} \lambda}{4 \pi \sigma_{x ; l}}\right)^{4}+\mathrm{BW}_{\mathrm{nl}}^{2}
\end{aligned}
$$

under the approximations already employed. The symbols $\langle A\rangle$ denote the statistical average of the variable $A$ and $\sigma_{A}^{2}$ its variance. We define

$$
\Psi_{0}=\gamma \theta_{\max } / \sqrt{1+X_{0}} .
$$

The recoil parameter reads

$$
X_{0}=2\left(1+\beta \cos \theta_{i}\right) E_{\lambda} E_{e} /\left(m_{e}^{2} c^{4}\right) .
$$

We also denote by $\epsilon_{n}$ the normalized electron beam transverse emittance. It is assumed identical in horizontal and vertical directions. The departure of the laser beam divergence from a pure Gaussian mode is included in the so-called beam quality factor $M^{2}$. The contribution of the nonlinearities [54] in the interaction are included thanks to the intensity parameter [55]

$$
a_{0}=6.8 \times 10^{-6} \frac{\lambda}{2 \sigma_{x ; l}} \sqrt{\frac{c U}{\sigma_{z ; l}}}
$$

in the term

$$
\mathrm{BW}_{\mathrm{nl}}^{2}=\left(\frac{a_{0}^{2} / 3}{1+a_{0}^{2} / 2}\right)^{2}
$$

This term stems from classical calculations [26].

We complement the work that has been previously done by the authors of Ref. [11] by adding an additional observable, the average energy of the photon beam that is obtained in a given aperture. Despite an accurate estimation is not really necessary at design stage, because the energy of the beam needs to be experimentally precisely tuned, for instance for NRF experiments, it however consists in an interesting figure of merit to judge the accuracy of the expression that are derived. It is moreover a necessary ingredient that enters in the denominator of the bandwidth $\frac{\sigma_{E_{\gamma}}}{\left\langle E_{\gamma}\right\rangle}$. The average energy of the photon beam reads approximately, in absence of contributions from electron-beam energy spread or emittance and laser-beam angular divergence, laser spectrum or nonlinearities,

$$
\begin{aligned}
\left\langle E_{\gamma}\right\rangle= & \frac{E_{e} X_{0}}{24\left(1+X_{0}\right) \tilde{X}_{0}^{2}}\left(24 \tilde{X}_{0}^{2}-12 \Psi_{0}^{2} \tilde{X}_{0}^{2}\right. \\
& \left.+2 \Psi_{0}^{4} \tilde{X}_{0}\left(16+\left(\tilde{X}_{0}-2\right)\left(10+X_{0}\right)\right)\right),
\end{aligned}
$$

where $\tilde{X}_{0}=2+X_{0}\left(2+X_{0}\right)$.

One shall notice that in these expressions, the parameter $X_{0}$ naturally account for the crossing angle $\theta_{i}$ in between the laser and electron beams. We will now concentrate on a situation where the laser beam divergence, the electronbeam emittance, and energy spread are vanishing. We also consider long-enough chirp-free laser pulses to ensure that the laser spectrum is not too broad to significantly spoil the 
bandwidth but their length is still smaller than the Rayleigh range, such that the hourglass effect related to the laser beam is negligible. Similarly, as already mentioned, one assumes that the hourglass effect can be neglected for the electron beam. In that situation, the two dominant contributions to the photon beam bandwidth come from the aperture and the nonlinearities related to the laser intensity. The accuracy of the estimation of this latter contribution with an analytical model must be good enough to justify its use for design purposes. It must be emphasized that the electronbeam energy spread, the laser spectrum, and the laser beam divergence are directly contributing through usual error propagation since they enter in the scaling of the scattered photon energy parameterized with $X_{0}$. Indeed the dependence of the cross section exhibits a very smooth dependence on $X_{0}$ in the typical energy ranges considered in Compton sources for $\mathrm{x}$ ray or nuclear physics applications.

\section{IMPROVED ANALYTICAL MODEL INCLUDING NONLINEARITIES}

We introduce the substitutions of the Eqs. (16) and (18), due to an updated investigation of nonlinearities, in this section. In order to do so, one must recognize that the parameter $\eta^{2}$ depends on the exact location and time of the interaction in the region where the beams are crossing since the laser beam is being assumed to have a given spatiotemporal distribution. We thus first estimate an average value for this parameter but also its variance. Approximate analytical formula to include contributions due to several subsequent Compton scattering and dressing of the electron mass in the strong field are then derived. The fraction of photons produced at harmonics due to multiphoton absorption is also estimated. Finally, we conclude by updating the formula given in Eq. (16) with all contributions added. To the best of our knowledge, these approximate formula are not given in the literature.

In order to give confidence in the model we develop, we decide to compare the results with predictions obtained by means of simulating the interaction of the laser and electron beams with the event generator CAIN 2.42 [29]. This software code is well tested in the accelerator physics and particle physics community and has been initially designed for the simulation of strong field effects between electron and photon beams in high-energy colliders [57]. An accurate description of electron and laser beams along with the physics of their collisions is implemented. In particular, it must be noted that all the nonlinearities described further in this paper are embedded in this generator, depending on running options [29].

To perform this benchmark, we implement the parameters listed in Table I, where the laser beam energy is intently chosen to be relatively large, in order to facilitate the benchmark exercise such that other possibly contributing effects to the bandwidth are made negligible for small apertures. Compton sources designs exists with similar magnitude of laser pulse energy [3,58].
TABLE I. Electron, laser, and interaction region parameters for the numerical benchmark of the analytical expression derived in this paper. The electron-beam parameters are inspired from Refs. $[8,19]$. The beam parameters values refer to those at the Compton interaction point.

\begin{tabular}{|c|c|}
\hline Electron-beam parameter & Value \\
\hline Bunch charge, $\mathcal{Q}$ & $100 \mathrm{pC}$ \\
\hline Average beam energy, $E_{e}$ & $300 \mathrm{MeV}$ \\
\hline Bunch length $^{\mathrm{a}}, \sigma_{z, e}$ & $150 \mu \mathrm{m}$ \\
\hline Horizontal rms spot size, $\sigma_{x, e}$ & $6 \mu \mathrm{m}$ \\
\hline Vertical rms spot size ${ }^{\mathrm{b}}, \sigma_{y, e}$ & $6 \mu \mathrm{m}$ \\
\hline Normalized rms emittance, $\epsilon_{n}$ & $55 \mathrm{nmrad}$ \\
\hline Relative rms energy spread, $\sigma_{E_{e}} / E_{e}$ & $10^{-4}$ \\
\hline Laser-beam parameter & Value \\
\hline Central wavelength, $\lambda$ & $1030 \mathrm{~nm}$ \\
\hline Pulse duration ${ }^{\mathrm{c}}, \sigma_{t, l}$ & $1.5 \mathrm{ps}$ \\
\hline Horizontal rms spot size, $\sigma_{x, l}$ & $7.5 \mu \mathrm{m}$ \\
\hline Vertical rms spot size ${ }^{\mathrm{d}}, \sigma_{y, l}$ & $7.5 \mu \mathrm{m}$ \\
\hline Single pulse energy, $U$ & $1 \mathrm{~J}$ \\
\hline Departure to Gaussian beam, $M^{2}$ & 1.2 \\
\hline Interaction region parameter & Value \\
\hline Crossing angle, $\theta_{i}$ & $3^{\circ}$ \\
\hline
\end{tabular}

${ }^{\mathrm{a}} \mathrm{A}$ vanishing dispersion is assumed.

${ }^{\mathrm{b}} \mathrm{A}$ symmetric beam is assumed in the transverse plane.

${ }^{\mathrm{c}}$ The laser pulse is assumed Fourier limited, free of chirp.

${ }^{\mathrm{d}} \mathrm{A}$ symmetric beam is assumed in the transverse plane.

\section{A. Laser intensity as seen by the electron beam}

The effect of electron dressing in the strong field actually depends on the laser intensity seen by the electron at the space-time location of the interaction. We assume a fundamental Gaussian laser beam and a longitudinally Gaussian laser pulse of duration $\sigma_{t ; l}=\sigma_{z ; l} / c$, the laser power at the maximum density (peak power) reads $P_{0}=U /\left(\sqrt{2 \pi} \sigma_{t ; l}\right)$. The peak and average intensity thus read $I_{\max }=2 P_{0} /\left(4 \pi \sigma_{x ; l} \sigma_{y ; l}\right)=2 P_{0} /\left(4 \pi \sigma_{x ; l}^{2}\right)$ and $\bar{I}=P_{0} /$ $\left(4 \pi \sigma_{x ; l}^{2}\right)$, respectively. Divergence effects are neglected here. We further define the quantity $\bar{\eta}^{2}=3.65 \times$ $10^{-19} \bar{I}\left[\mathrm{~W} / \mathrm{cm}^{2}\right] \lambda[\mu \mathrm{m}]^{2}$.

The laser intensity parameter seen by electrons in average $\widehat{\eta^{2}}$ slightly differs from the naively expected value $\bar{\eta}^{2}$. Indeed the electron beam itself carries a transverse distribution which will slightly increase the average laser intensity parameter that must be accounted for in the calculations. This average laser intensity parameter is actually related to the luminosity term by a similar integral over the four-dimensional space-time. One thus writes

$\widehat{\eta^{2}}=\frac{\int \eta^{2}\left(x_{L}, y_{L}, z_{L}, t\right) n_{e}(x, y, z, t) n_{l}\left(x_{L}, y_{L}, z_{L}, t\right) d x d y d z d t}{\int n_{e}(x, y, z, t) n_{l}\left(x_{L}, y_{L}, z_{L}, t\right) d x d y d z d t}$, 
where

$n_{e}(x, y, z, t)=\frac{\exp \left(-x^{2} / 2 \sigma_{x ; e}^{2}-y^{2} / 2 \sigma_{y ; e}^{2}-(z-c t)^{2} / 2 \sigma_{z ; e}^{2}\right)}{(2 \pi)^{3 / 2} \sigma_{x ; e} \sigma_{y ; e} \sigma_{z ; e}}$

is the electron-beam charge density,

$$
n_{l}\left(x_{L}, y_{L}, z_{L}, t\right)=\frac{\exp \left(-\frac{x_{L}^{2}}{2 \sigma_{x ; l}^{2}}-\frac{y_{L}^{2}}{2 \sigma_{y ; l}^{2}}-\frac{\left(z_{L}-c t\right)^{2}}{2 \sigma_{z ; l}^{2}}\right)}{(2 \pi)^{3 / 2} \sigma_{x ; l} \sigma_{y ; l} \sigma_{z ; l}}
$$

the laser-beam density with

$$
\begin{aligned}
& x_{L}=-x \cos \theta_{i}+z \sin \theta_{i}, \\
& z_{L}=-z \cos \theta_{i}-x \sin \theta_{i},
\end{aligned}
$$

and $y_{L}=y$. The laser intensity parameter reads

$\eta^{2}\left(x_{L}, y_{L}, z_{L}, t\right)=2 \bar{\eta}^{2} \exp \left(-\frac{x_{L}^{2}}{2 \sigma_{x ; l}^{2}}-\frac{y_{L}^{2}}{2 \sigma_{y ; l}^{2}}-\frac{\left(z_{L}-c t\right)^{2}}{2 \sigma_{z ; l}^{2}}\right)$.

It provides

$$
\begin{aligned}
\widehat{\eta^{2}}= & \frac{\bar{\eta}^{2}}{\sqrt{2}} \frac{\mathcal{L}_{0}\left(\sigma_{x ; l} / \sqrt{2}, \sigma_{y ; l} / \sqrt{2}, \sigma_{z ; l} / \sqrt{2}\right)}{\mathcal{L}_{0}\left(\sigma_{x ; l}, \sigma_{y ; l}, \sigma_{z ; l}\right)} \\
= & \sqrt{2} \bar{\eta}^{2} \frac{\sqrt{\sigma_{x, e}^{2}+\sigma_{x ; l}^{2}+\left(\sigma_{z ; e}^{2}+\sigma_{z ; l}^{2}\right) \tan ^{2}\left(\frac{\theta_{i}}{2}\right)}}{\sqrt{2 \sigma_{x ; e}^{2}+\sigma_{x ; l}^{2}+\left(2 \sigma_{z ; e}^{2}+\sigma_{z ; l}^{2}\right) \tan ^{2}\left(\frac{\theta_{i}}{2}\right)}} \\
& \times \frac{\sqrt{\sigma_{y, e}^{2}+\sigma_{y, l}^{2}}}{\sqrt{2 \sigma_{y, e}^{2}+\sigma_{y ; l}^{2}}} .
\end{aligned}
$$

The variance $\sigma_{\eta^{2}}^{2}=\left\langle\eta^{4}\right\rangle-\left\langle\eta^{2}\right\rangle$ of $\eta^{2}$ can be computed similarly to its expected value $\left\langle\eta^{2}\right\rangle=\widehat{\eta^{2}}$. It provides

$$
\begin{aligned}
\sigma_{\eta^{2}}^{2}= & \bar{\eta}^{4} \frac{4}{3 \sqrt{3}} \frac{\mathcal{L}_{0}\left(\sigma_{x, l} / \sqrt{3}, \sigma_{y, l} / \sqrt{3}, \sigma_{z, l} / \sqrt{3}\right)}{\mathcal{L}_{0}\left(\sigma_{x, l}, \sigma_{y, l}, \sigma_{z, l}\right)}-\left(\widehat{\eta^{2}}\right)^{2} \\
= & \bar{\eta}^{4} \frac{4}{\sqrt{3}} \frac{\sqrt{\sigma_{x, e}^{2}+\sigma_{x, l}^{2}+\left(\sigma_{z, e}^{2}+\sigma_{z, l}^{2}\right) \tan ^{2}\left(\frac{\theta_{i}}{2}\right)}}{\sqrt{3 \sigma_{x, e}^{2}+\sigma_{x, l}^{2}+\left(3 \sigma_{z, e}^{2}+\sigma_{z, l}^{2}\right) \tan ^{2}\left(\frac{\theta_{i}}{2}\right)}} \\
& \times \frac{\sqrt{\sigma_{y, e}^{2}+\sigma_{y, l}^{2}}}{\sqrt{3 \sigma_{y, e}^{2}+\sigma_{y, l}^{2}}}-\left(\widehat{\eta}^{2}\right)^{2} .
\end{aligned}
$$

The approximate magnitude of the luminosity reduction due to the neglected hourglass effect can be estimated precisely analytically [53] or numerically. The impact of the hourglass effect on the effective laser intensity parameter is indeed reduced since $\widehat{\eta^{2}}$ contains a ratio of luminosity terms. We estimated numerically that this ratio is well predicted by the ratio of approximate luminosities $\mathcal{L}_{0}$ within $10^{-4}$ in the parameter range considered in this article.

\section{B. Plural, subsequent, scatterings}

When the interaction region is sufficiently long, an electron that already scattered has a significant probability to interact a second time (or more) with a photon of the laser beam. The number of resulting photons gets slightly nonlinear in laser intensity. This effect has been studied by solving numerically the Chapman-Kolmogorov equation [59]. A more approximate but practical approach consists in assuming that an electron can interact at most twice, which was numerically found to be fair in the parameter range studied in this paper, and that we believe is appropriate in the design of an ultimate ICS. This approach was already employed in Ref. [41]. The typical mean free path of an electron in a relatively intense laser reads approximately $L=1 /\left(\sigma_{C} \rho_{I}\right)$ where

$$
\begin{gathered}
\sigma_{C}=\int_{0}^{\tilde{Y}_{\max }} \frac{d \sigma_{1}}{d \tilde{Y}} d \tilde{Y} \approx \sigma_{C, 0}+\widehat{\eta}^{2} \sigma_{C, \eta^{2}}, \quad \text { with } \\
\sigma_{C, 0}=\frac{2 \pi r_{e}^{2}}{X_{0}}\left(\left(1-\frac{4}{X_{0}}-\frac{8}{X_{0}^{2}}\right) \log \left(1+X_{0}\right)\right. \\
\left.+\frac{1}{2}+\frac{8}{X_{0}}-\frac{1}{2\left(1+X_{0}\right)^{2}}\right), \quad \text { and } \\
\sigma_{C, \eta^{2}}=\frac{2 \pi r_{e}^{2}}{X_{0}}\left(\frac{5}{X_{0}}-1+\frac{3+6 X_{0}+2 X_{0}^{2}}{X_{0}\left(1+X_{0}\right)^{3}}\right. \\
\left.-\frac{8 \log \left(1+X_{0}\right)}{X_{0}^{2}}\right)
\end{gathered}
$$

is the total Compton cross section where the modification of the electron mass due to the strong-field related to the laser beam is included up to the first order in $\eta^{2}$. The differential cross section is given by $\frac{d \sigma_{1}}{d \check{Y}}=\frac{d \sigma_{1}^{\text {circ }}}{d \tilde{Y}}=\frac{d \sigma_{1}^{\text {lin }}}{d \check{Y}}$ in Eqs. (7) or (11). The average density of photons is denoted $\rho_{I}=\hat{I} /\left(E_{\lambda} c\right)$, with $\hat{I}=\bar{I} \widehat{\eta}^{2} / \bar{\eta}^{2}$ the average laser intensity experienced by the electron beam expressed in $W / \mathrm{m}^{2}$. The typical interaction length of the laser beam reads $2 \sigma_{L}$, with $\sigma_{L}=\sqrt{\sigma_{z ; l}^{2} \cos ^{2} \theta_{i}+\sigma_{x ; l}^{2} \sin ^{2} \theta_{i}}$. When dealing with picosecond pulse durations, transverse laser beam size of less than couple of tens of micrometers and crossing angle of few degrees the interaction length approximately reduces to $2 \sigma_{z, l}$. The interaction probability of an electron is thus $p_{\text {int }}=1-\exp \left(-2 \sigma_{L} / L\right)$. When one considers reaching sub-percent bandwidths, this small effect must be accounted for [3]. Indeed electrons that already have interacted once will carry a smaller energy. The average energy of an electron after a first scattering reads $E_{e}\left(1-\delta_{E_{e}, p}\right)$, where 


$$
\begin{aligned}
\delta_{E_{e}, p} & =\int_{0}^{\tilde{Y}_{\max }} \frac{\tilde{Y}}{\sigma_{C}} \frac{d \sigma_{1}}{d \tilde{Y}} d \tilde{Y} \\
& \approx \frac{X_{0}}{40}\left(20-22 X_{0}-20 \widehat{\eta^{2}}+47 X_{0} \widehat{\eta^{2}}\right),
\end{aligned}
$$

after expanding $\delta_{E_{e}, p}$ up to the first order in $X_{0}$ and $\eta^{2}$.

The transverse momentum of the electrons is also slightly modified, but this effect has a negligible impact since the electron recoil is itself small. The average energy of the scattered photon beam, including the plural electron scattering reads

$$
\begin{aligned}
\left\langle E_{\gamma, p}\right\rangle & =\left\langle E_{\gamma}\right\rangle\left(1-\delta E_{\gamma, p}\right), \quad \text { where } \\
\delta E_{\gamma, p} & \simeq 2 \frac{p_{\mathrm{int}}}{1+p_{\mathrm{int}}} \delta_{E_{e}, p} .
\end{aligned}
$$

In order to perform a benchmark of these expressions, the event generator CAIN is used assuming that linear Compton cross sections are used (i.e., only $n=1$ photon is absorbed and $\eta^{2}=0$ ). This is ensured by the LASERQED $\mathrm{NPH}=0$ command. We thus compare the simulation results, using the parameters of Table I with Eq. (26) where one sets $\widehat{\eta^{2}}=0$ in Eqs. (25) and (22) but not in $\rho_{I}$. The numerical implementation of this expression shows an excellent agreement with results obtained with a CAIN simulation, see Fig. 1 (top). The residual relative difference between the two curves is about $2 \times 10^{-4}$. The relative magnitude of the shift $\delta E_{\gamma, p}$ induced by plural scatterings in this simulated case amounts to about $-0.15 \%$ for a vanishing aperture.

The expected contribution to the photon beam bandwidth is two fold. The first, dominant, and contribution stems from the fact that electrons that are scattering twice have an average energy, at the instant of the second scatter, that is smaller than initially, and thus generates some spread of the parameter $X_{0}$. A second contribution is related to the energy spread $\sigma_{E_{e}, p}$ of the electrons that are scattering twice. At the same order as previous series expansions, the contribution to the photon beam bandwidth due to plural scatterings reads

$$
\begin{aligned}
\frac{\sigma_{E_{\gamma, p}}^{2}}{\left\langle E_{\gamma, p}\right\rangle^{2}} \simeq & \left(\frac{2+X_{0}}{1+X_{0}}\right)^{2}\left(\frac{p_{\text {int }}}{1+p_{\text {int }}} \frac{\sigma_{E_{e}, p}^{2}}{E_{e}^{2}\left(1-\delta_{E_{e}, p}\right)^{2}}\right. \\
& \left.+\frac{p_{\text {int }}}{\left(1+p_{\text {int }}\right)^{2}} \delta_{E_{e}, p}^{2}\right),
\end{aligned}
$$

where

$$
\frac{\sigma_{E_{e}, p}^{2}}{E_{e}^{2}\left(1-\delta_{E_{e}, p}\right)^{2}} \simeq \frac{X_{0}^{2}}{40}\left(4-9 \widehat{\eta^{2}}-4 X_{0}+13 X_{0} \widehat{\eta^{2}}\right)
$$

It may be recognized that Eq. (28) looks like the naive expectation $\frac{X_{0}^{2}}{12\left(1+X_{0}\right)^{2}}$ that can be drawn for this quantity by
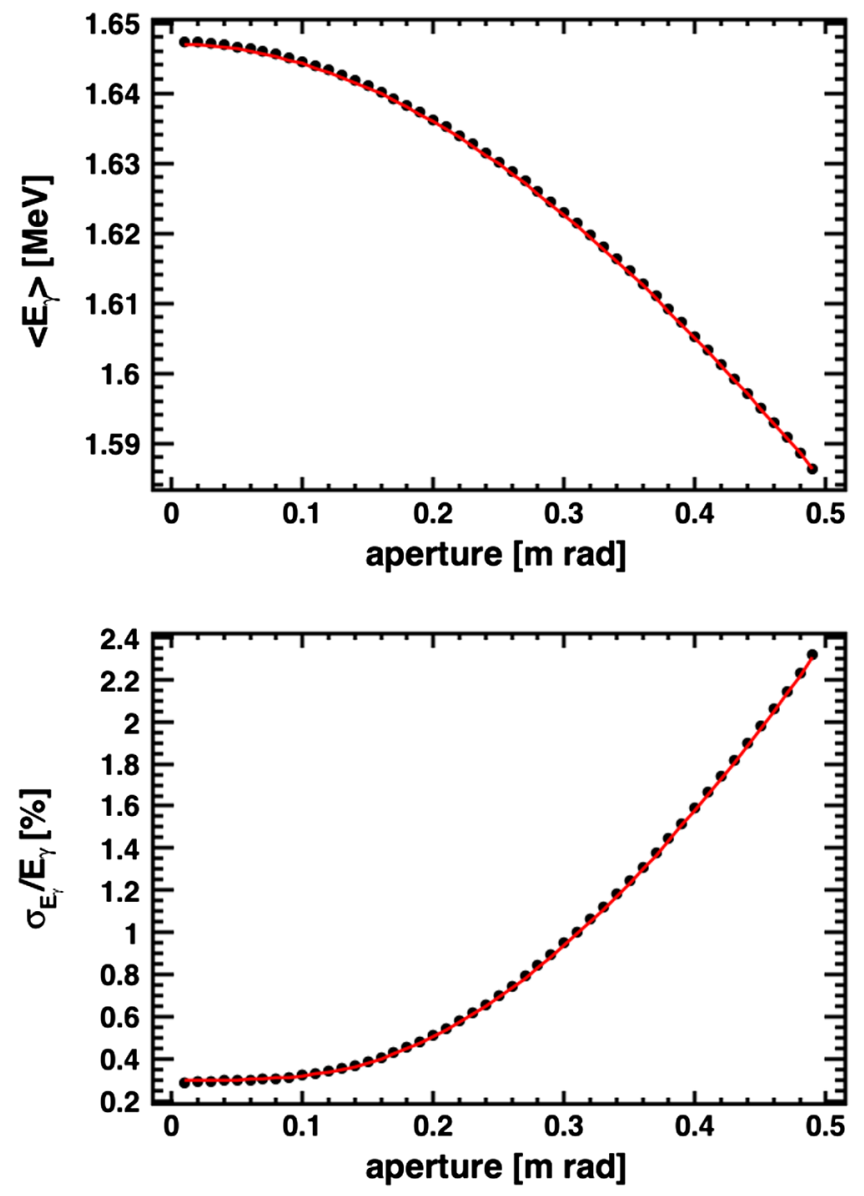

FIG. 1. Average energy (top) and relative bandwidth (bottom) of the photon beam estimated with CAIN (black dots) and using the analytical formula (red line) given in Eqs. (26) and (27) as a function of the angular aperture. The statistical uncertainty related to the finite statistics of the CAIN simulation is smaller that the size of the dots. The two curves are superimposed within a few $10^{-4}$.

assuming that the cross section is roughly flat and neglecting the modification of the cross section by strong field effects.

In order to benchmark this formula, one computes the photon bandwidth of Eq. (16) where $\mathrm{BW}_{\mathrm{nl}}^{2}$ is replaced by Eq. (27) with parameters of Table I. As previously done, one performs the replacement $\widehat{\eta^{2}} \rightarrow 0$ in the expression except in $\rho_{I}$ and compares these calculations with CAIN simulation results in linear regime. An excellent agreement is found, as shown in Fig. 1 (bottom). The behavior of the curve at low apertures is fully dominated by the contribution that we introduced here which amounts to about $0.3 \%$ with the chosen parameters.

\section{Effect of electron dressing}

Similarly to the reasoning performed for plural scattering, one computes the average energy shift of the produced photon beam due to the $\eta^{2}$ parameter that induces a 
modification of the electron mass in presence of strong electromagnetic fields. The average photon beam energy, neglecting the effect described in Sec. III, reads

$$
\left\langle E_{\gamma, d}\right\rangle=\left\langle E_{\gamma}\right\rangle\left(1-\delta E_{\gamma, d}\right)=\frac{\int_{\tilde{Y}_{\max }}^{\tilde{y}_{\max }} \tilde{Y} \frac{d \sigma_{1}}{d \tilde{Y}} d \tilde{Y}}{\int_{\tilde{Y}_{\min }}^{\tilde{Y}_{\max }} \frac{d \sigma_{1}}{d \tilde{Y}} d \tilde{Y}},
$$

where $\tilde{Y}_{\min }=\frac{X}{1+X}\left(1-\Psi^{2}+\Psi^{4}\right)$ and $\Psi=\gamma \theta / \sqrt{1+X}$. Expanding $\delta E_{\gamma, d}$ up to the second order in $X_{0}$, one obtains

$$
\delta E_{\gamma, d}=\frac{\widehat{\eta^{2}}}{\left(1+X_{0}\right)\left(1+\frac{\Psi_{0}^{2}}{2}\right)}-\frac{{\widehat{\eta^{2}}}^{2}}{\left(1+X_{0}\right)^{2}\left(1+\Psi_{0}^{2}\right)} .
$$

Since plural, subsequent, scatterings cannot be switched off while simulating the interaction using the CAIN program, we compare its results with the quantity $\left\langle E_{\gamma, d, p}\right\rangle=\left\langle E_{\gamma}\right\rangle\left(1-\delta E_{\gamma, p}\right)\left(1-\delta E_{\gamma, d}\right)$. It must be noted that, contrary to the previous section, now $\widehat{\eta^{2}}$ is not vanishing in the expressions used to compute $\delta E_{\gamma, p}$ since now dressing effects are considered. The effect of dressing amounts to few percent in the average energy shift and dominates over the contribution related to plural scatterings. The analytical formula and the result of the CAIN simulation with the single photon absorption allowed despite that $\eta^{2}$ is not vanishing anymore, LASERQED $\mathrm{NPH}=1$, match well according to Fig. 2 (top). The residual relative difference between the two curves is about $8 \times 10^{-4}$. Despite the agreement is slightly worse than for plural scattering it must be noted that not accounting for the electron mass shift would induce a disagreement of several percents. The residual difference between the model and the CAIN simulation cannot be attributed neither to the limited statistics of the Monte Carlo nor to a unaccounted hourglass effect according to our numerical investigations.

The main impact on the bandwidth is that the actual laser intensity seen by the electrons in the beam is not homogeneous. Since it contributes as a shift of the Compton threshold energy, the related contribution to the bandwidth of the photon beam reads

$$
\frac{\sigma_{E_{\gamma, d}}^{2}}{\left\langle E_{\gamma, d}\right\rangle^{2}}=\frac{\sigma_{\eta^{2}}^{2}}{\left(1-\widehat{\eta^{2}}\right)} \frac{\delta E_{\gamma, d}^{2}}{\widehat{\eta^{2}}} \approx \frac{\sigma_{\eta^{2}}^{2}}{\left(1+X_{0}\right)^{2}\left(1+\frac{\Psi_{0}^{2}}{2}\right)^{2}} .
$$

This contribution dominates that from the plural scattering, as can be seen in Fig. 2 (bottom). In this figure, the photon bandwidth is computed with Eq. (16) where $\mathrm{BW}_{\mathrm{nl}}^{2}=\frac{\sigma_{E_{\gamma, p}}^{2}}{\left\langle E_{\gamma, p}\right\rangle^{2}}+\frac{\sigma_{E_{\gamma, d}}^{2}}{\left\langle E_{\gamma, d}\right\rangle^{2}}$ as given by Eqs. (27) and (31).
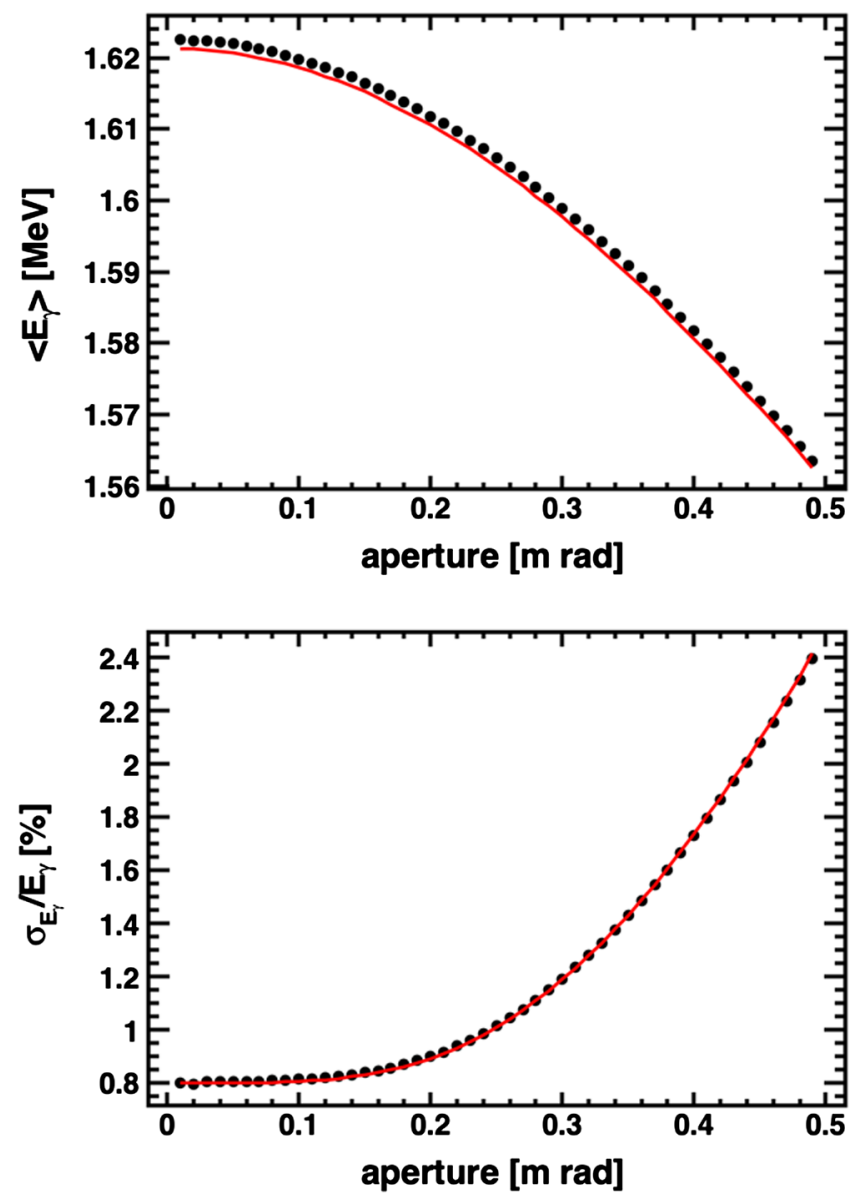

FIG. 2. Average energy (top) and relative bandwidth (bottom) computed using CAIN (black dots) and using the analytical formula (red line) given for plural scattering combined with effect related to electron dressing as a function of the angular aperture. The two curves are superimposed for most values of apertures.

\section{Effect of multiphoton absorption}

The last contribution that needs to be evaluated comes from multiple photon absorption. As already mentioned, one restrict ourselves to contributions from the second (circular and linear laser-beam polarization) and third (only in the case of linear laser-beam polarization) harmonics and neglect additional contributions related to higher orders, though they could be computed in a similar fashion. The average energy of the harmonic and the event rate in given apertures need to be calculated to asses their impact. This computation has to be done both for circular and linearly polarized laser beams. We define

$$
\left\langle E_{\gamma, n}^{\operatorname{circ}(\operatorname{lin})}\right\rangle=\int_{\tilde{Y}_{n, \text { min }}}^{\tilde{Y}_{n, \text { max }}} E_{e} \tilde{Y} \frac{d \sigma_{n}^{\operatorname{circ}(\operatorname{lin})}}{d \tilde{Y}} d \tilde{Y}
$$

and

$$
\left\langle\sigma_{n}^{\operatorname{circ}(\operatorname{lin})}\right\rangle=\int_{\tilde{Y}_{n, \text { min }}}^{\tilde{Y}_{n, \max }} \frac{d \sigma_{n}^{\operatorname{circ}(\operatorname{lin})}}{d \tilde{Y}} d \tilde{Y}
$$


where, $\tilde{Y}_{n, \min } \simeq \tilde{Y}_{n, \max }\left(1-\Psi^{2}+\Psi^{4}-\Psi^{6}\right), \tilde{Y}_{2, \max }=2 X /$ $(1+2 X)$, and $\tilde{Y}_{1, \max }=\tilde{Y}_{\max }$. As a matter of consequence, the Compton scattered photon beam will include several ensemble of photons of distinct energies. We show in Fig. 3 the spectrum of generated photons, with CAIN, with either purely circular or a purely linear laser beam polarization and otherwise identical conditions. The on axis third harmonic contribution strongly depends on the laser polarization. The effect of spectral broadening predominantly due to the strong field QED effect can be assessed on the bottom plot of this figure. A small contribution stemming from plural scatterings is also included.

The way harmonics need to be treated actually depends on the targeted applications of an ICS. The typical situation where ultimate bandwidths are required is that of NRF. One of the popular applications of this process is the nondestructive inspection of containers or cargo for nuclear nonproliferation purposes. In this situation, one of the techniques consists in placing a pure sample of the element that is being looked for after the container. In absence of this
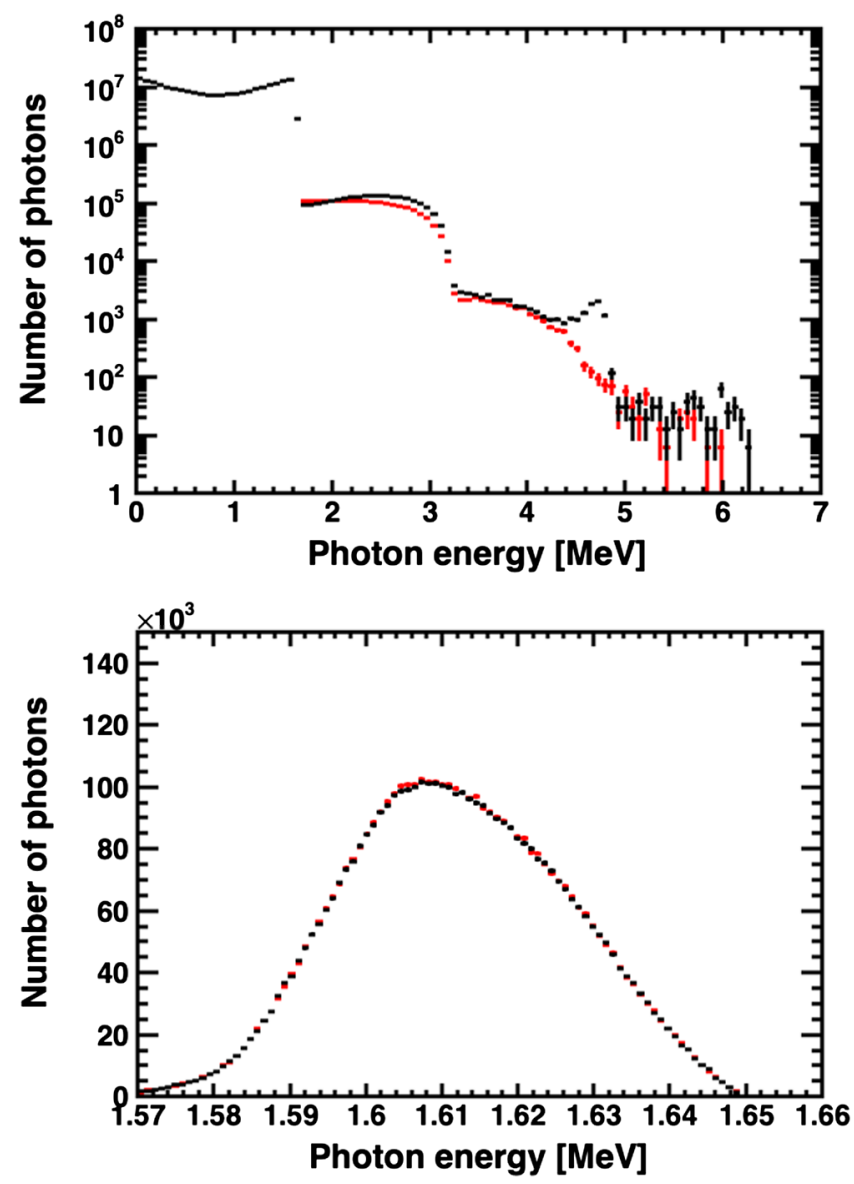

FIG. 3. Photon energy spectrum with all nonlinearities included for a circularly (linearly) polarized laser beam in red (black) in log-scale (top) and zoomed around the first order contribution within $200 \mu \mathrm{rad}$ aperture (bottom). On the latter the two distribution are hardly distinguishable by eye, as expected. element in the container, the detected NRF signal for the appropriate photon beam energy will be large. While if the element is abundant in the container, the photons of the beam at the NRF resonance of the element will interact prior impinging the test sample. As a matter of consequence, the detected NRF signal will be reduced. The depth of this notch in the NRF signal will depend on the abundance of the element in the container but also of backgrounds. However, photons with energies higher than the targeted element NRF energy may be scattered in the container itself and populate the narrow range of the element's NRF energy at the sample. The dip in the signal can thus be filled in by these higher energy photons produced in the ICS. This effect is often referred to as the notch refilling problem [7]. The fraction of photons that will refill the notch however depends on the details of the implementation of such an experiment. Experimental investigation of this effect seems to show that it is not a crippling issue [60].

Thus, we provide here estimates for the fraction of photons that are emitted at harmonic energies vs that at the fundamental. We believe having analytical formula for this quantity is helpful to asses the quality of the ICS for a specific application. We decompose the photon beam into several nearly monochromatic beams of different energies. At the first order in $\eta^{2}, X_{0}$, and second order in $\Psi_{0}^{2}$, the ratio of cross sections read

$$
\begin{gathered}
\frac{\left\langle\sigma_{2}^{\text {circ }}\right\rangle}{\left\langle\sigma_{1}^{\text {circ }}\right\rangle} \approx 4 \Psi_{0}^{2} \widehat{\eta^{2}}\left(1+X_{0}\right)\left(1-2 \Psi_{0}^{2}\right) \\
\frac{\left\langle\sigma_{2}^{\operatorname{lin}}\right\rangle}{\left\langle\sigma_{1}^{\operatorname{lin}}\right\rangle} \approx \Psi_{0}^{2} \widehat{\eta^{2}} \frac{1+X_{0}}{2}\left(13-25 \widehat{\eta^{2}}\right) \\
+\Psi_{0}^{4} \widehat{\eta^{2}}\left(-\frac{45}{2}+\frac{277}{6} \widehat{\eta^{2}}-X_{0}\left(\frac{283}{6}+\frac{337}{2} \widehat{\eta^{2}}\right)\right),
\end{gathered}
$$

and

$$
\begin{aligned}
\frac{\left\langle\sigma_{3}^{\operatorname{lin}}\right\rangle}{\left\langle\sigma_{1}^{\operatorname{lin}}\right\rangle} \approx & \frac{9\left(\widehat{\eta}^{2}\right)^{2}}{64}\left(-12\left(-1+3 X_{0}\right)\left(1+X_{0}+13 X_{0}^{2}\right)\right. \\
& +\Psi_{0}^{4}\left(728+X_{0}\left(2996+\left(2424-1061 X_{0}\right) X_{0}\right)\right) \\
& \left.-6 \Psi_{0}^{2}\left(18+X_{0}\left(22+X_{0}\left(-8+9 X_{0}\right)\right)\right)\right) .
\end{aligned}
$$

For small apertures, it is noteworthy that for circularly polarized laser beams, the second harmonic contribution scales approximately quadratically with the aperture. The fraction of photons can reach a few percent in extreme cases as for a $1 \mathrm{~J}$ laser beam and few hundreds of $\mu$ rad apertures.

The fraction of the number of photons emitted at these harmonic energies match that obtained with CAIN with LASERQED NPH=5 and the option LINEARPOL or CIRCULARPOL depending on the laser-beam polarization. The limited statistics in the harmonics is clearly seen in Fig. 4 at very small apertures. The aperture dependence of the fraction of photon is consistent with CAIN simulation within a few percents for values that is the range of 


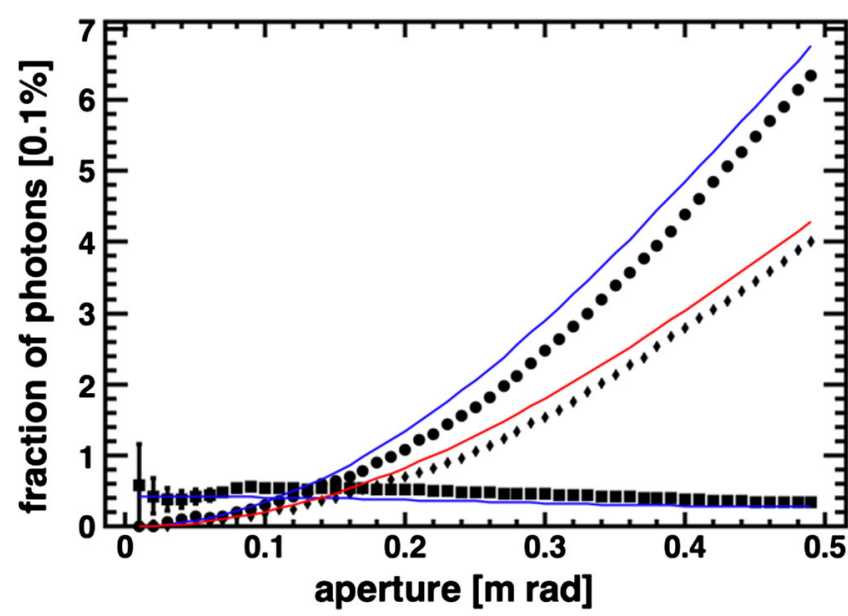

FIG. 4. The fraction of photons for the second (quadratic curves) and third (nearly linear curves) harmonics for purely circularly (red line and black diamonds) and linearly (blue lines and black dots and squared) polarized laser beams using the analytical formula (blue and red solid lines), and CAIN simulations (black markers).

few per-mille. This relative inacurracy may be explained by the approximations that were made while expanding the cross section and integrating those over ranges of interest. It must be noted that while scanning the aperture, the values, and statistical uncertainties of neighboring points are highly correlated since only a small number of events are additionally selected or removed. We however think that the analytical expressions proposed here are useful to have an immediate assessment of the quality of an ICS, in particular when looking at the optimization of such a source at the design stage.

\section{E. Discussion of the introduced model}

In the situation investigated in this article, where electron beams of an excellent emittance and energy spread are supposed to be used, effects related to the laser intensity may be relevant. The nonlinear broadening is found to be the dominant contributor to the bandwidth increase. It is sometimes considered in the literature that this effect can be compensated by means of an appropriate chirp of the laser field, see for instance Refs. [33,61-65]. Indeed, a chirp proportional to the laser intensity would compensate for the increased mass of the electron in the strong laser field. However, it is in practice extremely difficult to realize since this chirp must be done spatially and longitudinally to have a perfect compensation, despite arbitrary chirp may nowadays be realized [66], it needs to be applied to a specific laser system that is intended to be used for the ICS that one is designing. Finally, the relative magnitude of the Gaussian chirp, for a Gaussian laser pulse considered in this section must be of about $3 \%$, which thus requires to have a sufficient bandwidth in the laser system that is not necessarily guaranteed. Since, to the best of our knowledge, this technique has never been implemented in an actual ICS, we prefer to ignore this possibility in the present work.

As a summary, we suggest that at the design stage of an ICS targeting sub-percent photon beam bandwidth, Eq. (16) must be modified with Eqs. (27) and (31) by replacing the term in $a_{0}^{2}$ of Eq. (17). Similarly, Eq. (18) can be complemented by Eqs. (26) and (30) when needed, for instance to ensure that sufficient margin is accounted for when designing the maximal electron beam energy of the accelerator. The level of backgrounds due to harmonics can be assessed with Eqs. (34)-(36). The interest in these quantities is given in Sec. III. These modified expressions can be used to optimize an ICS, which is done in Sec. V.

We investigated that the estimates of bandwidth obtained with the analytical model proposed here is valid with a relative precision of a few percents even for $\bar{\eta}^{2}$ reaching about 0.15 , as shown on Fig. 5 . This has been obtained by increasing the laser pulse energy while keeping other parameters fixed. The model described in Ref. [11] is found to overestimate significantly nonlinear contributions, while the model developed in this article is found to match CAIN simulations.

In a similar fashion, we varied the energy of the electron beam to investigate the validity of the approximations used for the analytical model as function of the value of $X_{0}$. Here, the analytical model relatively underestimates the bandwidth by few $\%$ for $X_{0} \approx 0.016$, see Fig. 6 . Since this figure is made for a $1 \mathrm{~J}$ laser pulse, the discrepancy is expected to be much smaller at energies for which an ultimate bandwidth of about 1 per-mille can be reached. Overall the agreement is excellent in the parameter space that one expects to scan in the design of an ultimate ICS.

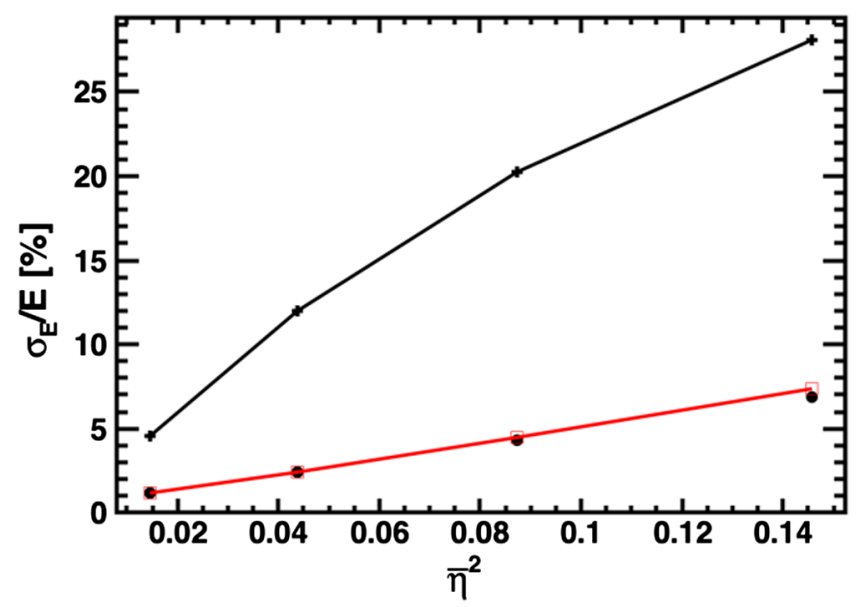

FIG. 5. Relative bandwidth of the photon beam estimated with CAIN (black dots), with the model proposed in this article (open squares) and the model of Ref. [11] (black crosses) as function of $\bar{\eta}^{2}$. Lines are drawn as a further guide to the eye for the analytical models. The laser pulse energy is varied from 1 to $10 \mathrm{~J}$ to realize this study. An aperture of $300 \mu \mathrm{rad}$ has been used to compute the relative bandwidth. 


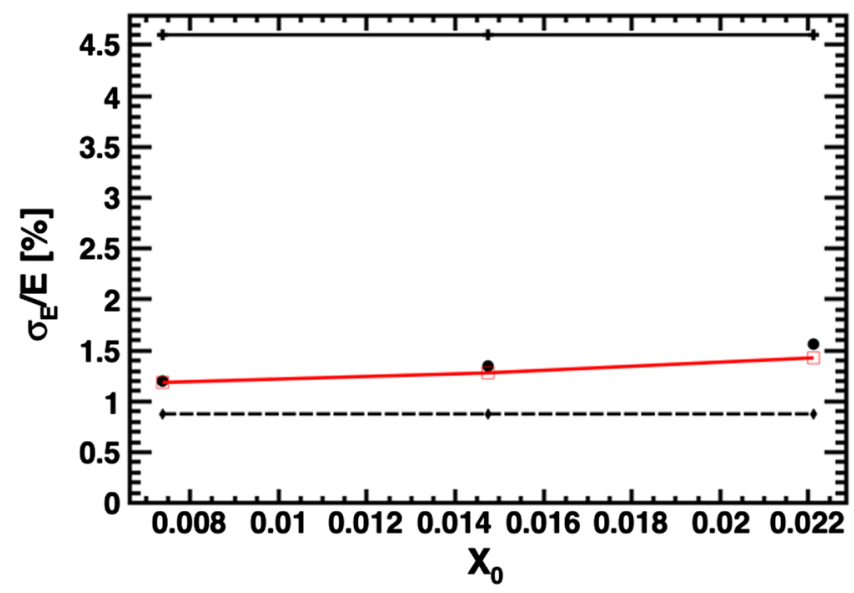

FIG. 6. Relative bandwidth of the photon beam estimated with CAIN (black dots), with the model proposed in this article (open red squares, red line), the model of Ref. [11] (black crosses, black line) and the same without the nonlinear term (black diamonds, dashed line) as function of $X_{0}$. Lines are drawn as a further guide to the eye for the analytical models. The electron beam energy is varied from 300 to $900 \mathrm{MeV}$ to realize this study. In order to be insensitive to the scaling of aperture $\theta_{\max }$ with energy, $\Psi_{0}$ is kept constant by choosing $\theta_{\max }=300 \mu \mathrm{rad}, 150 \mu \mathrm{rad}$, and $100 \mu \mathrm{rad}$ for $300 \mathrm{MeV}, 600 \mathrm{MeV}$, and $900 \mathrm{MeV}$, respectively.

Improving the accuracy of the analytical model could be done by increasing the order in the expansion in $X_{0}$, also for the terms in Eq. (16), which presents no significant difficulty but would provide longer expressions.

\section{INVESTIGATION OF DESIGNS DRIVEN BY PER-MILLE BANDWIDTH REQUIREMENTS}

Following the benchmarking realized in Sec. IV, we use the updated model for ICS optimization. We consider two typical cases here. When NRF experiments are expected to be done with such a $\gamma$-ray source, it is interesting to look at two figures of merit that must be coherently dealt with. The spectral density, as defined in Ref. [11], quantifies the number of photons in a useful energy range that is typically of $1 \mathrm{eV}$ accounting for Doppler broadening in the target material. Complementary to that figure of merit, background in the experiment will be proportional to the bandwidth of the photon beam produced by the source. The proportionality coefficients for these two figures of merit depend on the experimental settings. In case of cargo inspection or assay of nuclear materials $[67,68]$ it will depend on the shape of the container, the materials contained in it and the nucleus that is being investigated. The performance of the detection system as a whole will also affect the determination of these proportionality constants. Studies of a detailed implementation is out of the scope of this article. Thus at the preliminary design stage of an ICS for NRF applications, we simply look at curves of spectral density vs bandwidth to assess the performance of the source. Spectral densities shown in the figures of this section must be understood as per bunch crossing, and thus must be multiplied by an effective repetition rate to compute number of photons of interest produced per unit of time.

\section{A. Burst mode linacs}

The first case we study is that of a burst mode linac [8] coupled to a optical system able to deliver synchronous and aligned trains of laser pulses, for instance by means of laser pulse circulating device [24] or by use of a resonant FabryPerot cavity operated in burst mode [22,23]. While the former may deliver several hundreds of $\mathrm{mJ}$ of laser pulse energies recirculated about 30 times, the latter deliver slightly lower single pulse energies but with high-repetition rates and is more favorable when a large number of pulses in the train are required. Present day laser and optics manufacturing technology may allow to reach a hundred of $\mathrm{mJ}$ per pulse inside the optical cavity.

We assume here beam parameters identical to those given in Table I except that we vary the laser-beam transverse and longitudinal size. The minimally achievable bandwidth of the photon beam, defined as the bandwidth obtained for a vanishing aperture, is shown in Fig. 7 for three different assumptions of laser pulse energy and for a given laser beam transverse size of $\sigma_{x(y), l}=7.5 \mu \mathrm{m}$. It is compared with that obtained with formula of Ref. [11] for a $25 \mathrm{~mJ}$ laser beam and shows that it generally overestimates the bandwidth estimation except when the pulse duration is large enough such that contribution related to the crossing angle in Eq. (21) becomes important in the new formula introduced in this work. In the situation depicted here, a bandwidth of $0.1 \%$ cannot be reached with $100 \mathrm{~mJ}$ laser

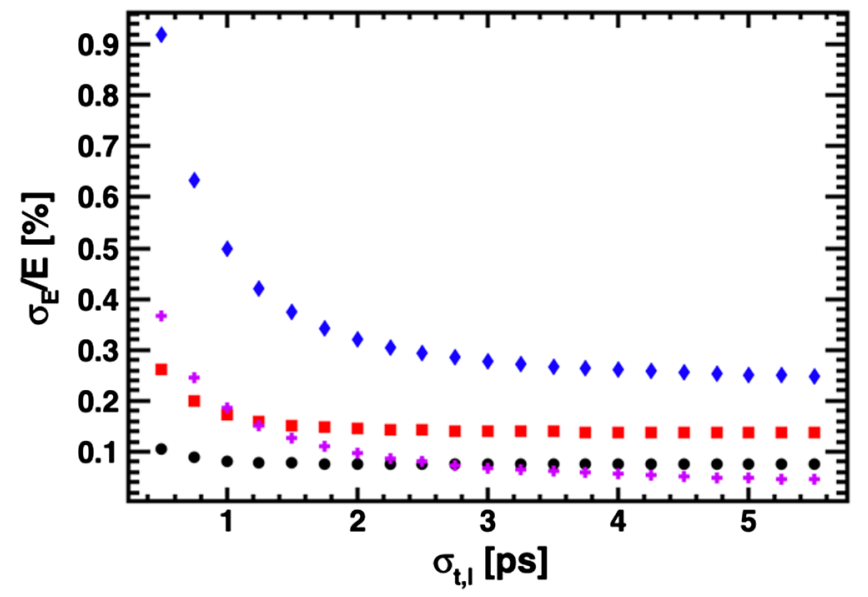

FIG. 7. The minimal achievable bandwidth (for a vanishing aperture) as function of the laser beam pulse duration, for a transverse size of $\sigma_{x, y, l}=7.5 \mu \mathrm{m}$ in both horizontal and vertical directions and laser beam single pulse energies of $25 \mathrm{~mJ}$ (black dots), $100 \mathrm{~mJ}$ (red squares), and $400 \mathrm{~mJ}$ (blue diamonds). The similar curve with formula of Ref. [11] for a $25 \mathrm{~mJ}$ laser beam is given with pink crosses. 


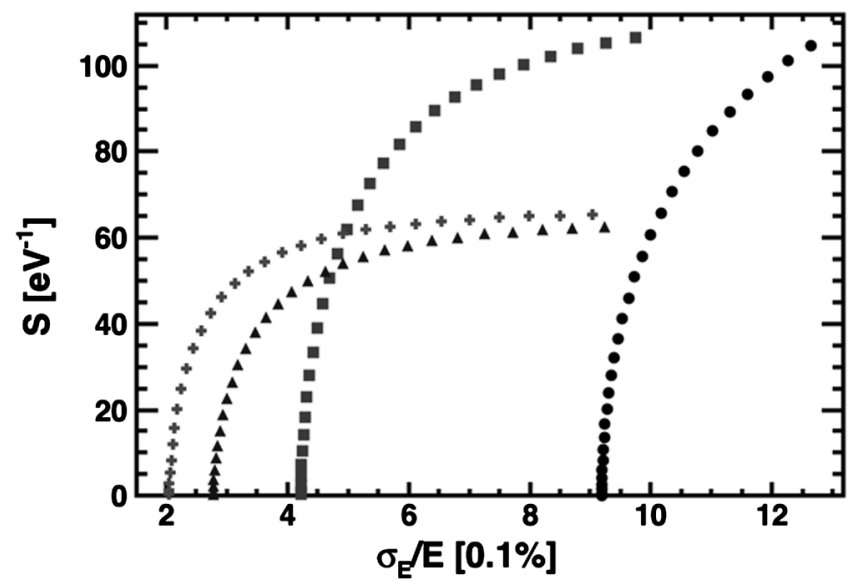

FIG. 8. The spectral density vs the ICS bandwidth for a laser pulse energy of $U=400 \mathrm{~mJ}$, (black dots) $\sigma_{t ; l}=0.5 \mathrm{ps}$, and $\sigma_{x ; l}=\sigma_{y ; l}=7.5 \mu \mathrm{m}$, (gray squares) $\sigma_{t ; l}=1.25 \mathrm{ps}$ and $\sigma_{x ; l}=$ $\sigma_{y ; l}=7.5 \mu \mathrm{m}$, (black triangles) $\sigma_{t ; l}=3 \mathrm{ps}$ and $\sigma_{x ; l}=\sigma_{y ; l}=$ $7.5 \mu \mathrm{m}$, and (gray crosses) $\sigma_{t ; l}=1.25 \mathrm{ps}$ and $\sigma_{x ; l}=$ $\sigma_{y ; l}=12.5 \mu \mathrm{m}$.

pulses, as was proposed for instance in Ref. [8]. The performance of the ICS can be further scrutinized in the spectral density vs bandwidth plane for various laser pulse duration and laser beam transverse sizes at the interaction point and for two laser beam single pulse energies of $400 \mathrm{~mJ}$ in Fig. 8 and $25 \mathrm{~mJ}$ in Fig. 9. These curves are obtained by varying the aperture. The optimal aperture will depend on details related to the experiments that would be performed with the ICS under consideration. It is however interesting to note that it is more profitable to increase the transverse laser beam size while decreasing the laser pulse duration. Indeed it will tend to increase the useful photon

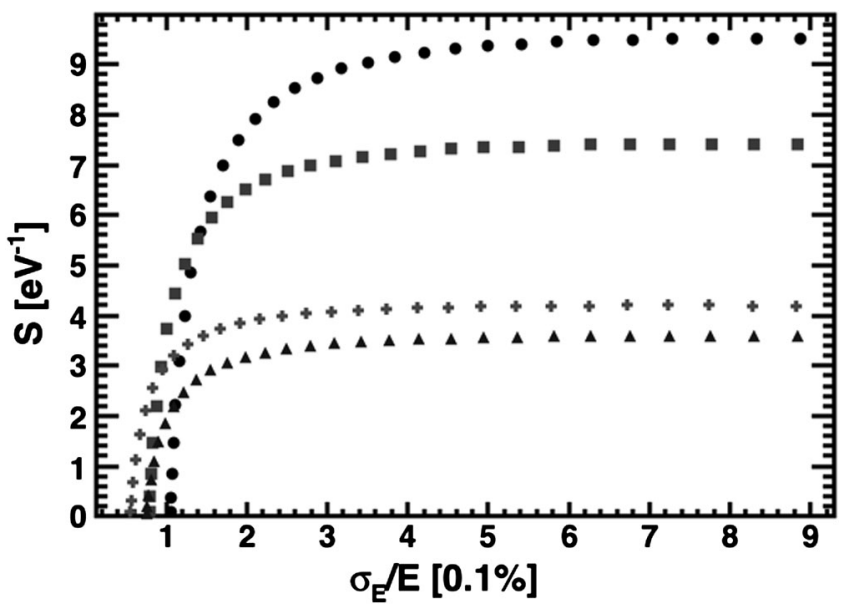

FIG. 9. The spectral density vs the ICS bandwidth for a laser pulse energy of $U=25 \mathrm{~mJ}$, (black dots) $\sigma_{t, l}=0.5$ ps and $\sigma_{x, l}=$ $\sigma_{y, l}=7.5 \mu \mathrm{m}$, (gray squares) $\sigma_{t, l}=1.25 \mathrm{ps}$ and $\sigma_{x, l}=\sigma_{y, l}=$ $7.5 \mu \mathrm{m}$, (black triangles) $\sigma_{t, l}=3 \mathrm{ps}$ and $\sigma_{x, l}=\sigma_{y, l}=7.5 \mu \mathrm{m}$, and (gray crosses) $\sigma_{t, l}=1.25 \mathrm{ps}$ and $\sigma_{x, l}=\sigma_{y, l}=12.5 \mu \mathrm{m}$.

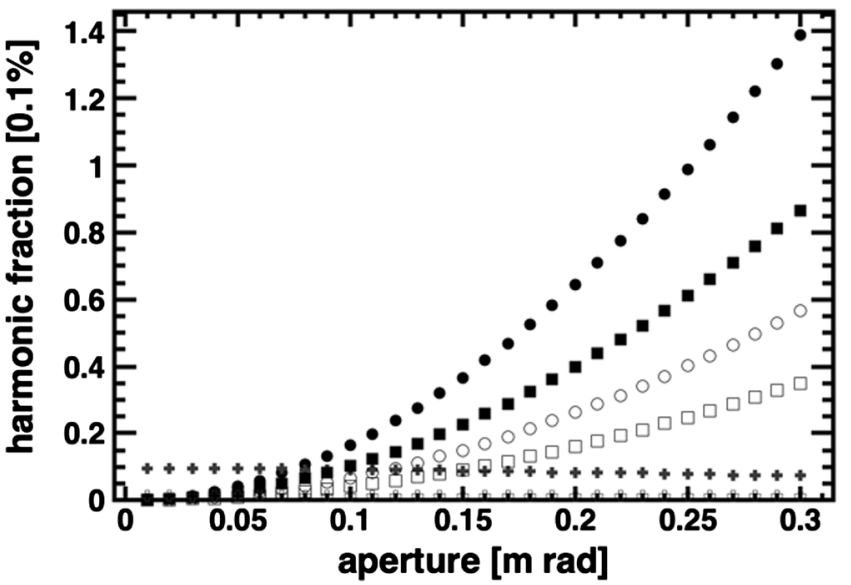

FIG. 10. The fraction of photons emitted on the second harmonic (black increasing quadratic curves with aperture) and third harmonic (gray linear decreasing curves with aperture) for a laser beam with pure circular (full and open squares) or linear (full and open dots or crosses) polarization as function of aperture. They are shown for a $U=400 \mathrm{~mJ}$ pulse of $\sigma_{t, l}=$ $1.25 \mathrm{ps}$ and $\sigma_{x, l}=7.5 \mu \mathrm{m}$ (full markers), and $\sigma_{x, l}=12.5 \mu \mathrm{m}$ (open markers). The third harmonic contribution is negligible in the $\sigma_{x, l}=12.5 \mu \mathrm{m}$ case.

yield (the spectral density) while reducing the background levels (the bandwidth). This is due to the predominant Piwinski contribution to the luminosity term and similar terms in the effective variance of the laser intensity, as shown in Eq. (21). We complement these observations by providing the fraction of photons that are emitted at the second harmonic (in circular and linear laser beam polarization) and third harmonic (for linear laser beam polarization only). These are shown for a $U=400 \mathrm{~mJ}$ pulse of $\sigma_{t, l}=1.25 \mathrm{ps}$ and $\sigma_{x, l}=7.5(12.5) \mu \mathrm{m}$ in Fig. 10. If judged as an issue, these curves can be used to optimize laser-beam parameters according to the requirements.

\section{B. Energy recovery linacs}

We investigate another popular type of ICS $[20,21]$ based on the energy recovery linac technology. One assumes here the parameters listed in Table II, in particular an energy spread of $10^{-3}$ and a normalized RMS emittance of $1 \mathrm{~mm}$ mrad.

We observe that with such a type of source, given the larger emittance and energy spread generally expected $[20,21]$, the effect of nonlinearities due to the laser-beam energy is mostly washed out as shown on Fig. 11. It must be noted that the $25 \mathrm{~mJ}$ single pulse laser energy is already corresponding to a total power of $1 \mathrm{MW}$ inside the optical cavity, for this particular project that uses a repetition frequency of $40 \mathrm{MHz}$. This figure is beyond the existing state of the art [69]. We note that the typical bandwidth that can be obtained is similar (slightly smaller) to that of the EuroGammaS proposal for a linac-based source [3] with a smaller spectral density essentially due to a reduced bunch 
TABLE II. Electron, laser, and interaction region parameters for the ERL-based ICS investigated in this paper. The beam parameters values are quoted at the Compton interaction point.

\begin{tabular}{|c|c|}
\hline Electron-beam parameter & Value \\
\hline Bunch charge, $\mathcal{Q}$ & $100 \mathrm{pC}$ \\
\hline Average beam energy, $E_{e}$ & $500 \mathrm{MeV}$ \\
\hline Bunch length $^{\mathrm{a}}, \sigma_{z ; e}$ & $900 \mu \mathrm{m}$ \\
\hline Horizontal rms spot size, $\sigma_{x ; e}$ & $30 \mu \mathrm{m}$ \\
\hline Vertical rms spot size ${ }^{\mathrm{b}}, \sigma_{y ; e}$ & $30 \mu \mathrm{m}$ \\
\hline Normalized rms emittance, $\epsilon_{n}$ & $1 \mathrm{~mm} \mathrm{mrad}$ \\
\hline Relative rms energy spread, $\sigma_{E_{e}} / E_{e}$ & $10^{-3}$ \\
\hline
\end{tabular}

\begin{tabular}{lr}
\hline \hline Laser-beam parameter & Value \\
\hline Central wavelength, $\lambda^{\mathrm{c}} \lambda$ & $1030 \mathrm{~nm}$ \\
Pulse duration $^{\mathrm{N}}, \sigma_{t ; l}$ & $3 \mathrm{ps}$ \\
${\text { Horizontal rms spot size, } \sigma_{x ; l}}_{\text {Vertical rms spot size }^{\mathrm{d}}, \sigma_{y ; l}}^{15 \mu \mathrm{m}}$ \\
Single pulse energy, $U^{\text {Departure to Gaussian beam, } M^{2}}$ & $15 \mu \mathrm{m}$ \\
& $25 \mathrm{~mJ}$ \\
\hline \hline
\end{tabular}

Interaction region parameter

Value

Crossing angle, $\theta_{i}$

${ }^{\mathrm{a}} \mathrm{A}$ vanishing dispersion is assumed.

${ }^{\mathrm{b}} \mathrm{A}$ symmetric beam is assumed in the transverse plane.

${ }^{\mathrm{c}}$ The laser pulse is assumed Fourier limited, free of chirp.

${ }^{\mathrm{d}} \mathrm{A}$ symmetric beam is assumed in the transverse plane.

charge and laser pulse energy. However due to the $40 \mathrm{MHz}$ repetition rate with $\mathrm{CW}$ duty cycle, this source would surpass the existing proposal of Ref. [3]. Both this technology and that of burst mode linacs, discussed in the Sec. IV, where effective collision rates of about a

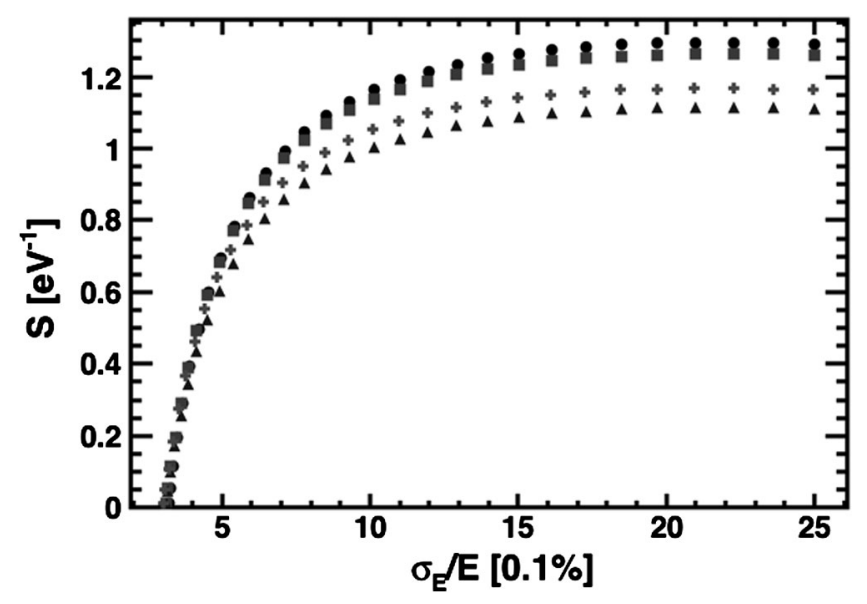

FIG. 11. The spectral density vs the ERL ICS bandwidth for a laser pulse energy of $U=25 \mathrm{~mJ}$, (black dots) $\sigma_{t, l}=0.5 \mathrm{ps}$ and $\sigma_{x, l}=\sigma_{y, l}=7.5 \mu \mathrm{m}$, (gray squares) $\sigma_{t, l}=1.25 \mathrm{ps}$ and $\sigma_{x, l}=$ $\sigma_{y, l}=7.5 \mu \mathrm{m}$, (black triangles) $\sigma_{t, l}=3 \mathrm{ps}$ and $\sigma_{x, l}=\sigma_{y, l}=$ $7.5 \mu \mathrm{m}$, and (gray crosses) $\sigma_{t, l}=1.25 \mathrm{ps}$ and $\sigma_{x, l}=\sigma_{y, l}=$ $12.5 \mu \mathrm{m}$. million per second (with few thousand bunches and about a $\mathrm{kHz}$ repetition rate system) are competing in terms of timeaverage spectral density i.e., useful photons delivered to experiments over a second. Very low bandwidth can only be reached with the burst mode linacs, which shows that this technology is actually expected to be superior. This conclusion would not hold anymore if the brightness of the considered ERLs could be significantly improved [70].

\section{CONCLUSION}

We have shown that a quantum viewpoint can be interestingly used to revisit the impact of nonlinearities on the spectral purity of high-energy photon sources based on Compton scattering. We derived analytical expressions that are benchmarked against a well established event generator for Compton scattering of photons from a laser beam off a beam of electrons. We further employed these expressions to optimize a few laser-beam parameters of ICS for either linacs operated in burst mode or superconducting ERLs. We finally show that the impact of the laser-beam energy on the brightness of the photon source is significant only in the case of an electron beam of excellent quality.

\section{ACKNOWLEDGMENTS}

The authors are thankful to Camilla Curatolo, Walid Kaabi, James Rozensweig, Yusuke Sakai, Luca Serafini, and Alessandro Variola for fruitful discussions.

[1] H. R. Weller, M. W. Ahmed, H. Gao, W. Tornow, Y. K. Wu, M. Gai, and R. Miskimen, Prog. Part. Nucl. Phys. 62, 257 (2009).

[2] K. Aoki, K. Hosono, T. Hadame, H. Munenaga, K. Kinoshita, M. Toda, S. Amano, S. Miyamoto, T. Mochizuki, M. Aoki, and D. Li, Nucl. Instrum. Methods Phys. Res., Sect. A 516, 228 (2004).

[3] O. Adriani et al., arXiv:1407.3669.

[4] Z. Pan, J. Byrd, H. Hao, W. Huang, D. Li, C. Sun, Y. K. $\mathrm{Wu}$, and C. Tang, Phys. Rev. Accel. Beams 22, 040702 (2019).

[5] C. R. Howell et al., arXiv:2012.10843.

[6] I. V. Pogorelsky, M. Polyanskiy, and T. Shaftan, Phys. Rev. Accel. Beams 23, 120702 (2020).

[7] J. Pruet, D. P. McNabb, C. A. Hagmann, F. V. Hartemann, and C. P. J. Barty, J. Appl. Phys. 99, 123102 (2006).

[8] W. S. Graves, J. Bessuille, P. Brown, S. Carbajo, V. Dolgashev, K.-H. Hong, E. Ihloff, B. Khaykovich, H. Lin, K. Murari, E. A. Nanni, G. Resta, S. Tantawi, L. E. Zapata, F. X. Kärtner, and D. E. Moncton, Phys. Rev. ST Accel. Beams 17, 120701 (2014).

[9] F. V. Hartemann, F. Albert, C. W. Siders, and C. P. J. Barty, Phys. Rev. Lett. 105, 130801 (2010).

[10] G. A. Krafft and G. Priebe, Rev. Accel. Sci. Techol. 03, 147 (2010).

[11] C. Curatolo, I. Drebot, V. Petrillo, and L. Serafini, Phys. Rev. Accel. Beams 20, 080701 (2017). 
[12] N. Ranjan, B. Terzić, G. A. Krafft, V. Petrillo, I. Drebot, and L. Serafini, Phys. Rev. Accel. Beams 21, 030701 (2018).

[13] M. Jacquet and C. Bruni, J. Synchrotron Radiat. 24, 312 (2017).

[14] Z. Huang and R. D. Ruth, Phys. Rev. Lett. 80, 976 (1998).

[15] I. Chaikovska et al., Sci. Rep. 6, 36569 (2016).

[16] I. Chaikovska and A. Variola, Phys. Rev. ST Accel. Beams 17, 044004 (2014).

[17] T. Akagi, A. Kosuge, S. Araki, R. Hajima, Y. Honda, T. Miyajima, M. Mori, R. Nagai, N. Nakamura, M. Shimada, T. Shizuma, N. Terunuma, and J. Urakawa, Phys. Rev. Accel. Beams 19, 114701 (2016).

[18] L. Piersanti et al., in Proceedings in 7th Particle Accelerator Conference (IPAC'16), Busan, Korea (JACoW, Geneva, 2016), pp. 407-410.

[19] J. Rosenzweig, A. Cahill, B. Carlsten, G. Castorina, M. Croia, C. Emma, A. Fukusawa, B. Spataro, D. Alesini, V. Dolgashev, M. Ferrario, G. Lawler, R. Li, C. Limborg, J. Maxson, P. Musumeci, R. Pompili, S. Tantawi, and O. Williams, Nucl. Instrum. Methods Phys. Res., Sect. A 909, 224 (2018).

[20] L. Serafini et al., Nucl. Instrum. Methods Phys. Res., Sect. A 930, 167 (2019).

[21] D. Angal-Kalinin et al., J. Phys. G 45, 065003 (2018).

[22] K. Sakaue, M. Washio, S. Araki, M. Fukuda, Y. Honda, N. Terunuma, and J. Urakawa, Rev. Sci. Instrum. 89, 023305 (2018).

[23] P. Favier, L. Amoudry, K. Cassou, R. Chiche, K. Dupraz, A. Martens, D. Nutarelli, V. Soskov, F. Zomer, A. Courjaud, and L. Serafini, Phys. Rev. Accel. Beams 21, 121601 (2018).

[24] C. F. Ndiaye et al., Phys. Rev. Accel. Beams 22, 093501 (2019).

[25] Y. Y. Lau, F. He, D. P. Umstadter, and R. Kowalczyk, Phys. Plasmas 10, 2155 (2003).

[26] V. Petrillo, A. Bacci, R. B. Al Zinati, I. Chaikovska, C. Curatolo, M. Ferrario, C. Maroli, C. Ronsivalle, A. Rossi, L. Serafini, P. Tomassini, C. Vaccarezza, and A. Variola, Nucl. Instrum. Methods Phys. Res., Sect. A 693, 109 (2012)

[27] F. Hartemann, High-Field Electrodynamics (CRC Press, Boca Raton, 2002), https://www.routledge.com/High-FieldElectrodynamics/Hartemann/p/book/9780849323782.

[28] G. A. Krafft, Phys. Rev. Lett. 92, 204802 (2004).

[29] K. Yokoya, Users manual of cain; version. 2.35, 2003.

[30] D. Seipt, Strong-field QED processes in short laser pulses. One- and two-photon Compton scattering, Ph.D. thesis, Technische University Dresden (Germany), Inst. fuer Theoretische Physik, Germany, 2012.

[31] G. A. Krafft, E. Johnson, K. Deitrick, B. Terzić, R. Kelmar, T. Hodges, W. Melnitchouk, and J. R. Delayen, Phys. Rev. Accel. Beams 19, 121302 (2016).

[32] J. M. Krämer, A. Jochmann, M. Budde, M. Bussmann, J. P. Couperus, T. E. Cowan, A. Debus, A. Köhler, M. Kuntzsch, A. L. García, U. Lehnert, P. Michel, R. Pausch, O. Zarini, U. Schramm, and A. Irman, Sci. Rep. 8, 1398 (2018).

[33] B. Terzić, A. Brown, I. Drebot, T. Hagerman, E. Johnson, G. A. Krafft, C. Maroli, V. Petrillo, and M. Ruijter, Europhys. Lett. 126, 12003 (2019).
[34] A. Denner and S. Dittmaier, Nucl. Phys. B540, 58 (1999).

[35] M. L. Swartz, Phys. Rev. D 58, 014010 (1998).

[36] H. Veltman, Phys. Rev. D 40, 2810 (1989).

[37] H. Veltman, Phys. Rev. D 42, 1856 (1990).

[38] von W. Heitler and L. Nordheim, Physica (Utrecht) 1, 1059 (1934).

[39] F. Mandl and T. H. R. Skyrme, Proc. R. Soc. A 215, 497 (1952).

[40] L. M. Brown and R. P. Feynman, Phys. Rev. 85, 231 (1952).

[41] C. Bamber, S. J. Boege, T. Koffas, T. Kotseroglou, A. C. Melissinos, D. D. Meyerhofer, D. A. Reis, W. Ragg, C. Bula, K. T. McDonald, E. J. Prebys, D. L. Burke, R. C. Field, G. Horton-Smith, J. E. Spencer, D. Walz, S. C. Berridge, W. M. Bugg, K. Shmakov, and A.W. Weidemann, Phys. Rev. D 60, 092004 (1999).

[42] Y. T. Grinchishin and M. P. Rekalo, Sov. J. Nucl. Phys. 40, 115 (1984).

[43] M. V. Galynskii and S. Sikach, Zh. Eksp. Teor. Fiz. 101, 828 (1992) [Sov. Phys. JETP 74, 441 (1992)].

[44] Y. S. Tsai, Phys. Rev. D 48, 96 (1993).

[45] J. H. Eberly, in Progress in Optics, edited by E. Wolf (Elsevier, New York, 1969), pp. 359-415, https://doi.org/ 10.1016/S0079-6638(08)70598-5.

[46] A. Hartin, Second order QED processes in an intense electromagnetic field, Ph.D. thesis, University of London Queen Mary, Department of Physics, 2017, arXiv:1701 .02906 .

[47] V. Berestetskii, E. Lifshitz, and L. Pitaevskii, in Quantum Electrodynamics (Second Edition), 2nd ed., edited by V. Berestetskii, E. Lifshitz, and L. Pitaevskii (ButterworthHeinemann, Oxford, 1982), pp. 354-455.

[48] K. D. Shmakov, Study of nonlinear QED effects in interactions of terawatt laser with high-energy electron beam, Ph.D. thesis, The University of Tennessee, 1997, https:// www.slac.stanford.edu/pubs/slacreports/reports10/slac-r666.pdf.

[49] C. Curatolo, I. Drebot, V. Petrillo, and L. Serafini, Phys. Rev. Accel. Beams 20, 109901 (2017).

[50] R. Hajima, Nucl. Instrum. Methods Phys. Res., Sect. A 985, 164655 (2021).

[51] T. Suzuki, General formulas of luminosity for various types of colliding beam machines, Technical Report No. KEK76-3, 1976.

[52] The term effective is employed to designate the fact that bunches are not necessarily evenly separated in time, but can be grouped in trains that are repeated at a relatively low frequency in time.

[53] M. Furman, The Hourglass reduction factor for asymmetric colliders, Technical Report Nos. SLAC-ABC-41-REV, ESG-NOTE-161, 1991.

[54] D. D. Meyerhofer, IEEE J. Quantum Electron. 33, 1935 (1997).

[55] There is apparently a mismatch of definition of the $a_{0}^{2}$ parameter defined in Ref. [11] that is a factor $2.5 \approx \sqrt{2 \pi}$ larger than the peak laser intensity parameter usually employed [56] and a factor five larger than the $\bar{\eta}^{2}$ parameter used throughout the paper. For consistency with the existing literature on the very specific subject dealt with in this article, we keep the value of $a_{0}$ as defined in Ref. [11]. 
[56] E. Esarey, C. B. Schroeder, and W. P. Leemans, Rev. Mod. Phys. 81, 1229 (2009).

[57] P. Chen, G. Horton-Smith, T. Ohgaki, A. Weidemann, and K. Yokoya, Nucl. Instrum. Methods Phys. Res., Sect. A 355, 107 (1995).

[58] A. Bacci et al., in Proceedings of the International Particle Accelerator Conference (IPAC 16), Busan, Korea, 2016, (JACoW, Geneva, Switzerland, 2016), International Particle Accelerator Conference No. 7, pp. 1747-1750.

[59] V. Petrillo, A. Bacci, C. Curatolo, C. Maroli, L. Serafini, and A. R. Rossi, J. Appl. Phys. 114, 043104 (2013).

[60] C. A. Hagmann, J. M. Hall, M. S. Johnson, D. P. McNabb, J. H. Kelley, C. Huibregtse, E. Kwan, G. Rusev, and A. P. Tonchev, J. Appl. Phys. 106, 084901 (2009).

[61] D. Seipt, S. G. Rykovanov, A. Surzhykov, and S. Fritzsche, Phys. Rev. A 91, 033402 (2015).

[62] C. Maroli, V. Petrillo, I. Drebot, L. Serafini, B. Terzi, and G. A. Krafft, J. Appl. Phys. 124, 063105 (2018).

[63] I. Ghebregziabher, B. A. Shadwick, and D. Umstadter, Phys. Rev. ST Accel. Beams 16, 030705 (2013).
[64] B. Terzic, K. Deitrick, A. S. Hofler, and G. A. Krafft, Phys. Rev. Lett. 112, 074801 (2014).

[65] B. Terzic, C. Reeves, and G. A. Krafft, Phys. Rev. Accel. Beams 19, 044403 (2016).

[66] C. E. Rogers III, M. J. Wright, J. L. Carini, J. A. Pechkis, and P. L. Gould, J. Opt. Soc. Am. B 24, 1249 (2007).

[67] R. Hajima, T. Hayakawa, N. Kikuzawa, and E. Minehara, J. Nucl. Sci. Technol. 45, 441 (2008).

[68] W. Bertozzi and R. J. Ledoux, Nucl. Instrum. Methods Phys. Res., Sect. B 241, 820 (2005).

[69] H. Carstens, N. Lilienfein, S. Holzberger, C. Jocher, T. Eidam, J. Limpert, A. Tünnermann, J. Weitenberg, D. C. Yost, A. Alghamdi, Z. Alahmed, A. Azzeer, A. Apolonski, E. Fill, F. Krausz, and I. Pupeza, Opt. Lett. 39, 2595 (2014).

[70] C. Gulliford, A. Bartnik, I. Bazarov, L. Cultrera, J. Dobbins, B. Dunham, F. Gonzalez, S. Karkare, H. Lee, H. Li, Y. Li, X. Liu, J. Maxson, C. Nguyen, K. Smolenski, and Z. Zhao, Phys. Rev. ST Accel. Beams 16, 073401 (2013). 